\title{
Second-order Knudsen-layer analysis for the generalized slip- flow theory II: Curvature effects
}

$\operatorname{AUTHOR}(S)$ :

Hattori, Masanari; Takata, Shigeru

\section{CITATION:}

Hattori, Masanari ...[et al]. Second-order Knudsen-layer analysis for the generalized slipflow theory II: Curvature effects. Journal of Statistical Physics 2015, 161(4): 1010-1036

\section{ISSUE DATE:}

2015

URL:

http://hdl.handle.net/2433/261188

\section{RIGHT:}

This is a post-peer-review, pre-copyedit version of an article published in Journal of Statistical Physics. The final authenticated version is available online at: http://dx.doi.org/10.1007/s10955-015-1364-0.; This is not the published version. Please cite only the published version.; この論文は出版社版でありません。引用の際には出版社版をご碓認じ 利用ください。 
Journal of Statistical Physics manuscript No.

(will be inserted by the editor)

\title{
Second-Order Knudsen-Layer Analysis for the Generalized Slip-Flow Theory II: Curvature Effects
}

\author{
Masanari Hattori · Shigeru Takata
}

(Date of submission: May 29, 2015)

\begin{abstract}
Numerical analyses of the second-order Knudsen layer are carried out on the basis of the linearized Boltzmann equation for hard-sphere molecules under the diffuse reflection boundary condition. The effects of the boundary curvature have been clarified in details, thereby completing the numerical data required up to the second order of the Knudsen number for the asymptotic theory of the Boltzmann equation (the generalized slip-flow theory). A local singularity appears as a result of the expansion at the level of the velocity distribution function, when the curvature exists.
\end{abstract}

Keywords Boltzmann equation, Kinetic theory, Slip flow, Curvature effect, Divergence singularity, Knudsen number, Rarefied gas

PACS 51.10.+y, 47.45.-n, 47.45.Ab, 47.45.Gx, 47.61.Cb

Mathematics Subject Classification (2010) 35Q20, 76P05, 76M45, $82 \mathrm{C} 40,82 \mathrm{D} 05$

\section{Introduction}

Study on the connection between the kinetic theory and the fluid-dynamics has a long history [1-3] and a number of important results have been obtained for a small or a vanishing limit of the Knudsen number, e.g., [4-9]. A systematic asymptotic theory has been established in the late 1960s and early 1970s $[4,5]$

M. Hattori

Department of Mechanical Engineering and Science, Kyoto University, Kyoto-daigakuKatsura, Kyoto 615-8540, Japan

S. Takata

Department of Aeronautics and Astronautics (Also at Advanced Research Institute of Fluid Science and Engineering), Kyoto University, Kyoto-daigaku-Katsura, Kyoto 615-8540, Japan E-mail: takata.shigeru.4a@kyoto-u.ac.jp 
and developed further [6] since then. It provides not only the fluid-dynamic description in the bulk region but also the slip/jump boundary condition and the non-fluid-like correction in a thin layer adjacent to the boundary (the Knudsen layer) for small Knudsen numbers. We call the linear case of that theory the generalized slip-flow theory in the present paper. The generalized slip-flow theory contains rich information on the gas rarefaction effect, giving a fluiddynamical interpretation to various phenomena occurring for small Knudsen numbers. It also motivates recent studies on time-dependent problems in its framework [10-12]. In the meantime, mathematical studies are also developing to include the argument of boundary condition for the fluid-dynamical equation [13-15].

The generalized slip-flow theory has been applied to various fundamental problems and was revealed to be practical. However, such applications have been limited mostly to the studies based on the Bhatnagar-Gross-Krook (BGK) [or Boltzmann-Krook-Welander (BKW)] model equation [16,17]. This is due to lack of numerical data of the Knudsen layer at the second order of the Knudsen number for the original Boltzmann equation. We have recently obtained the required numerical data up to the second-order of the Knudsen number [18], except for the effects of boundary curvature, assuming the hard-sphere molecules and the diffuse reflection boundary condition.

In the present paper, we take a step further to complete the full set of numerical data that are required in applications up to the second order of the Knudsen number expansion. They have been missing for nearly a half century in applying the theory to specific problems on the basis of the original Boltzmann equation. To be a little more specific, we focus on the effects of curvature of the boundary that manifest themselves at the second order of the Knudsen number. As will be clarified in the course of analyses, even though the macroscopic quantities are finite and well defined, the velocity distribution function diverges locally in the molecular velocity space in approaching or on the boundary. This feature forced us to separate the present work from the previous one [18]. The local but theoretical drawback behind the benefit of the expansion will also be discussed. We have developed the numerical method that handles such a singularity safely to complete the data required in the generalized slip-flow theory.

\section{Generalized Slip-Flow Theory: Outline and Present Concern}

Let us consider a gas around smooth solid bodies which do not change in time their shape and position under the following assumptions: (i) There is no external force and the behavior of the gas is described by the Boltzmann equation for monatomic molecules; (ii) The gas molecules are reflected locally isotropically [6] on the surface of the solid bodies (no net flow across their surface); (iii) The deviation from the reference equilibrium state at rest with density $\rho_{0}$ and temperature $T_{0}$ is so small that the equation and the initial and the boundary condition can be linearized around that equilibrium state; 
(iv) The mean free path $\ell_{0}$ of a molecule at the reference equilibrium state is much smaller than the characteristic length $L$ of the physical system (i.e., the Knudsen number $\left.\mathrm{Kn}=\ell_{0} / L \ll 1\right)$; (v) The time evolution is initiated by a slow change of the surroundings from the reference equilibrium state.

The time scale of the change $t_{0}$ in $(\mathrm{v})$ is the same order as that of the viscous and the thermal diffusion, and we set it as $t_{0}=(2 / \sqrt{\pi})\left(L^{2} / \ell_{0} \sqrt{2 R T_{0}}\right)$, where $R$ is the specific gas constant (the Boltzmann constant $k$ divided by the mass of a molecule $m$ ). In the actual computations for the Knudsen-layer analysis, we assume the hard-sphere molecules in (i) and the diffuse reflection boundary condition in (ii). For hard-sphere molecules, $\ell_{0}=\left[\sqrt{2} \pi d_{m}^{2}\left(\rho_{0} / m\right)\right]^{-1}$, where $d_{m}$ is the diameter of a molecule.

The generalized slip-flow theory consists of first considering the overall behavior of the gas that changes in the scale of the characteristic length (and time) of the system and then introducing the correction in the vicinity of the boundary. The first part is conducted by the Hilbert (or Grad-Hilbert) expansion to yield a set of fluid-dynamic equations. The solution of this set is called the Hilbert part (or solution). The second part is conducted by the expansion after stretching the spatial coordinate in the direction normal to the boundary. This yields a set of slip/jump boundary condition and the associated correction to the fluid-dynamic solution near the boundary. The correction is called the Knudsen-layer correction, which is the present primary concern.

We shall denote by $L x_{i}$ the space coordinates, by $t_{0} t$ the time, by $\rho_{0}(1+\omega)$ the density of the gas, by $\left(2 R T_{0}\right)^{1 / 2} u_{i}$ the flow velocity, by $T_{0}(1+\tau)$ the temperature, by $p_{0}(1+P)$ the pressure with $p_{0}=\rho_{0} R T_{0}$, and by $\left(2 R T_{0}\right)^{1 / 2} u_{i \mathrm{w}}$ and $T_{0}\left(1+\tau_{\mathrm{w}}\right)$ the velocity and the temperature of the body surface. Since solid bodies change neither the shape nor the position, $u_{i \mathrm{w}} n_{i}=0$, where $n_{i}$ is the unit vector normal to the surface, pointed to the gas. We denote the Hilbert part and the Knudsen-layer correction of the macroscopic quantity $h\left(h=\omega, u_{i}, \tau, P\right)$ by $h_{\mathrm{H}}$ and $h_{\mathrm{K}}$, respectively: $h=h_{\mathrm{H}}+h_{\mathrm{K}}$. Since the Knudsen number is small, we use $\varepsilon=(\sqrt{\pi} / 2) \mathrm{Kn}$ as a small parameter. The Hilbert part and the Knudsen-layer correction are obtained by a power series expansion in $\varepsilon$, which will be denoted as $h_{\mathrm{H}}=h_{\mathrm{H} 0}+h_{\mathrm{H} 1} \varepsilon+h_{\mathrm{H} 2} \varepsilon^{2}+\cdots$ and $h_{\mathrm{K}}=h_{\mathrm{K} 0}+h_{\mathrm{K} 1} \varepsilon+h_{\mathrm{K} 2} \varepsilon^{2}+\cdots$. Actually, the expansion of $h_{\mathrm{K}}$ starts from $O(\varepsilon)$, because no correction to the Hilbert solution is required at $O(1)$.

The resulting set of fluid-dynamic equations, their slip/jump boundary conditions, and the Knudsen-layer corrections up to the second order of the expansion in $\varepsilon$ are summarized as follows: [10]

Fluid-dynamic equations

$$
\begin{aligned}
& \frac{\partial P_{\mathrm{H} 0}}{\partial x_{i}}=0, \\
& \frac{\partial u_{i \mathrm{H} m}}{\partial x_{i}}+\frac{\partial \omega_{\mathrm{H} m-1}}{\partial t}=0, \\
& \frac{\partial u_{i \mathrm{H} m}}{\partial t}+\frac{1}{2} \frac{\partial P_{\mathrm{H} m+1}^{*}}{\partial x_{i}}-\frac{1}{2} \gamma_{1} \frac{\partial^{2} u_{i \mathrm{H} m}}{\partial x_{j}^{2}}+\frac{1}{4}\left(\gamma_{1} \gamma_{10}-2 \gamma_{6}\right) \frac{\partial^{4} u_{i \mathrm{H} m-2}}{\partial x_{j}^{2} \partial x_{k}^{2}}=0,
\end{aligned}
$$




$$
\begin{aligned}
& \frac{\partial \tau_{\mathrm{H} m}}{\partial t}-\frac{2}{5} \frac{\partial P_{\mathrm{H} m}}{\partial t}-\frac{1}{2} \gamma_{2} \frac{\partial^{2} \tau_{\mathrm{H} m}}{\partial x_{j}^{2}}+\frac{1}{10}\left(\gamma_{2} \gamma_{3}-\frac{13}{2} \gamma_{11}\right) \frac{\partial^{4} \tau_{\mathrm{H} m-2}}{\partial x_{j}^{2} \partial x_{k}^{2}}=0, \\
& P_{\mathrm{H} m+1}^{*}=P_{\mathrm{H} m+1}-\frac{1}{6}\left(\gamma_{2} \gamma_{1}-4 \gamma_{3}\right) \frac{\partial^{2} \tau_{\mathrm{H} m-1}}{\partial x_{j}^{2}}+\frac{1}{5} \gamma_{1} \frac{\partial P_{\mathrm{H} m-1}}{\partial t}, \\
& P_{\mathrm{H} m}=\omega_{\mathrm{H} m}+\tau_{\mathrm{H} m},
\end{aligned}
$$

where $m=0,1,2$ and the quantities $h_{\mathrm{H}-1}$ and $h_{\mathrm{H}-2}\left(h=\omega, u_{i}, \tau, P\right)$ should be read as zero. The $\gamma$ 's occurring in the equations are positive constants corresponding to the transport coefficients at the reference state; $\gamma$ 's are all unity for the BGK model, while they are respectively $\gamma_{1}=1.270042427, \gamma_{2}=$ $1.922284066, \gamma_{3}=1.947906335, \gamma_{6}=1.419423836, \gamma_{10}=1.63607346$, and $\gamma_{11}=2.7931173$ for hard-sphere molecules.

Slip/jump boundary condition and the Knudsen-layer correction

$$
\begin{aligned}
& {\left[\begin{array}{c}
\left(u_{i \mathrm{H} m}-u_{i \mathrm{w} m}\right) t_{i} \\
u_{i \mathrm{~K} m} t_{i}
\end{array}\right]=\frac{\overline{\partial u_{i \mathrm{H} m-1}}}{\partial x_{j}} n_{i} t_{j}\left[\begin{array}{c}
b_{1}^{(1)} \\
Y_{1}^{(1)}(\eta)
\end{array}\right]+\frac{\partial \tau_{\mathrm{H} m-1}}{\partial x_{i}} t_{i}\left[\begin{array}{c}
b_{2}^{(1)} \\
Y_{2}^{(1)}(\eta)
\end{array}\right]} \\
& +\frac{\partial^{2} \tau_{\mathrm{H} m-2}}{\partial x_{i} \partial x_{j}} n_{i} t_{j}\left[\begin{array}{c}
b_{3}^{(1)} \\
Y_{3}^{(1)}(\eta)
\end{array}\right]+\frac{\partial}{\partial x_{i}} \frac{\overline{\partial u_{j \mathrm{H} m-2}}}{\partial x_{k}} n_{i} n_{j} t_{k}\left[\begin{array}{c}
b_{4}^{(1)} \\
Y_{4}^{(1)}(\eta)
\end{array}\right] \\
& +\bar{\kappa} \frac{\overline{\partial u_{i \mathrm{H} m-2}}}{\partial x_{j}} n_{i} t_{j}\left[\begin{array}{c}
b_{5}^{(1)} \\
Y_{5}^{(1)}(\eta)
\end{array}\right]+\kappa_{i j} \frac{\overline{\partial u_{j \mathrm{H} m-2}}}{\partial x_{k}} n_{k} t_{i}\left[\begin{array}{c}
b_{6}^{(1)} \\
Y_{6}^{(1)}(\eta)
\end{array}\right] \\
& +\kappa_{i j} \frac{\partial \tau_{\mathrm{H} m-2}}{\partial x_{i}} t_{j}\left[\begin{array}{c}
b_{7}^{(1)} \\
Y_{7}^{(1)}(\eta)
\end{array}\right]+\bar{\kappa} \frac{\partial \tau_{\mathrm{H} m-2}}{\partial x_{i}} t_{i}\left[\begin{array}{c}
b_{8}^{(1)} \\
Y_{8}^{(1)}(\eta)
\end{array}\right], \\
& {\left[\begin{array}{l}
u_{i \mathrm{H} m} n_{i} \\
u_{i \mathrm{~K} m} n_{i}
\end{array}\right]=\frac{1}{2} \frac{\partial}{\partial x_{i}} \frac{\overline{\partial u_{j \mathrm{H} m-2}}}{\partial x_{k}} n_{i} n_{j} n_{k}\left[\begin{array}{c}
\int_{0}^{\infty} Y_{1}^{(1)}(z) d z \\
-\int_{\eta}^{\infty} Y_{1}^{(1)}(z) d z
\end{array}\right]} \\
& +\left[2 \bar{\kappa} \frac{\partial \tau_{\mathrm{H} m-2}}{\partial x_{i}} n_{i}-\frac{\partial^{2} \tau_{\mathrm{H} m-2}}{\partial x_{i} \partial x_{j}}\left(\delta_{i j}-n_{i} n_{j}\right)\right]\left[\begin{array}{c}
\int_{0}^{\infty} Y_{2}^{(1)}(z) d z \\
-\int_{\eta}^{\infty} Y_{2}^{(1)}(z) d z
\end{array}\right], \\
& {\left[\begin{array}{c}
\tau_{\mathrm{H} m}-\tau_{\mathrm{w} m} \\
\omega_{\mathrm{K} m} \\
\tau_{\mathrm{K} m}
\end{array}\right]=\frac{\partial \tau_{\mathrm{H} m-1}}{\partial x_{i}} n_{i}\left[\begin{array}{c}
c_{1}^{(0)} \\
\Omega_{1}^{(0)}(\eta) \\
\Theta_{1}^{(0)}(\eta)
\end{array}\right]+\frac{\partial u_{i \mathrm{H} m-1}}{\partial x_{i}}\left[\begin{array}{c}
c_{5}^{(0)} \\
\Omega_{5}^{(0)}(\eta) \\
\Theta_{5}^{(0)}(\eta)
\end{array}\right]} \\
& +\frac{\partial^{2} \tau_{\mathrm{H} m-2}}{\partial x_{i} \partial x_{j}}\left(\delta_{i j}-n_{i} n_{j}\right)\left[\begin{array}{c}
c_{2}^{(0)} \\
\Omega_{2}^{(0)}(\eta) \\
\Theta_{2}^{(0)}(\eta)
\end{array}\right]+\frac{\partial^{2} \tau_{\mathrm{H} m-2}}{\partial x_{j}^{2}}\left[\begin{array}{c}
c_{6}^{(0)} \\
\Omega_{6}^{(0)}(\eta) \\
\Theta_{6}^{(0)}(\eta)
\end{array}\right] \\
& +\frac{\partial}{\partial x_{i}} \frac{\overline{\partial u_{j \mathrm{H} m-2}}}{\partial x_{k}} n_{i} n_{j} n_{k}\left[\begin{array}{c}
c_{3}^{(0)} \\
\Omega_{3}^{(0)}(\eta) \\
\Theta_{3}^{(0)}(\eta)
\end{array}\right]+\bar{\kappa} \frac{\partial \tau_{\mathrm{H} m-2}}{\partial x_{i}} n_{i}\left[\begin{array}{c}
c_{4}^{(0)} \\
\Omega_{4}^{(0)}(\eta) \\
\Theta_{4}^{(0)}(\eta)
\end{array}\right],
\end{aligned}
$$

$P_{\mathrm{K} m}=\omega_{\mathrm{K} m}+\tau_{\mathrm{K} m}$, 
where $m=0,1,2$ and $\overline{f_{i j}}=f_{i j}+f_{j i}-(2 / 3) f_{k k} \delta_{i j}$ ( $\delta_{i j}$ is the Kronecker delta). In (2), the quantities with the subscript $\mathrm{H}$ or $\mathrm{w}$ denote their values at the (dimensionless) surface position $x_{i \mathrm{w}}$, and $t_{i}$ (or $n_{i}$ ) is a unit vector tangential (or normal) to the surface at $x_{i \mathrm{w}}$. The quantities with the subscript $\mathrm{K}$ depend on $\eta$ as well as $x_{i \mathrm{w}}$, where $\eta$ is the stretched spatial coordinate normal to the surface at $x_{i \mathrm{w}}$ such that the position $x_{i}$ in the Knudsen layer is expressed by $x_{i}=x_{i \mathrm{w}}+\varepsilon \eta n_{i}$. The surface velocity and temperature are also expanded in a power series of $\varepsilon: h_{\mathrm{w}}=h_{\mathrm{w} 0}+h_{\mathrm{w} 1} \varepsilon+h_{\mathrm{w} 2} \varepsilon^{2}+\cdots\left(h=u_{i}, \tau\right)$. The effect of surface curvature occurs through the terms with $\kappa$ 's defined by

$$
\bar{\kappa}=\frac{1}{2}\left(\kappa_{1}+\kappa_{2}\right), \quad \kappa_{i j}=\kappa_{1} \ell_{i} \ell_{j}+\kappa_{2} m_{i} m_{j} .
$$

Here $\kappa_{1} / L$ and $\kappa_{2} / L$ are the principal curvatures of the boundary, with $\kappa_{1}$ and $\kappa_{2}$ being taken negative when the corresponding center of curvature lies on the gas side; $\ell_{i}$ and $m_{i}$ are the direction cosines of the principal directions corresponding to $\kappa_{1}$ and $\kappa_{2}$ respectively. It is those terms that we are interested in in the present paper.

Remark 1 Many terms degenerate from (1) and (2) when $m=0,1$. For instance, the boundary condition at $O(1)$, namely for $m=0$, is none other than the non-slip/non-jump condition; the slip/jump may occur at the first or higher order of $\varepsilon$. The reader is referred to $[6,10]$ for the discussions on the main features of the above system. The system can be solved from the lowest order to determine the time-dependent behavior of the gas under the considered situation, provided that the data of the slip/jump coefficients $c_{1}^{(0)} \sim c_{6}^{(0)}, b_{1}^{(1)} \sim b_{8}^{(1)}$, $\int_{0}^{\infty} Y_{1}^{(1)}(z) d z, \int_{0}^{\infty} Y_{2}^{(1)}(z) d z$ and the elemental (or component) Knudsen-layer functions $\Omega_{1}^{(0)}(\eta) \sim \Omega_{6}^{(0)}(\eta), \Theta_{1}^{(0)}(\eta) \sim \Theta_{6}^{(0)}(\eta), Y_{1}^{(1)}(\eta) \sim Y_{8}^{(1)}(\eta)$ are available. They are obtained by solving elemental (or component) half-space problems of the linearized Boltzmann equation, which may be homogeneous or inhomogeneous depending on that component.

Since the first publication of the generalized slip-flow theory $[4,5]$, the complete set of the slip/jump coefficients and the associated Knudsen-layer corrections has been available only for the BGK model. For the original Boltzmann equation, a part of the information have been reported in the late 1980s and early 1990s [19-21], assuming the hard-sphere molecules and the diffuse reflection boundary condition. The present work reports the final step of our attempts that follows these and our recent works $[10,18,22]$. Thanks to the theory of symmetry relation [23], we have already obtained the complete set of slip/jump coefficients up to the second order of $\varepsilon$ in [10]. However, the information of the Knudsen-layer structure is still incomplete, which motivates the present work. As will become clear in the sequel, the numerical method developed in [18], which is based on the integral formulation of the Boltzmann equation [24,25], is really effective in the study of the Knudsen layer related to the curvature effects of boundary. 


\section{Knudsen-Layer Problems}

Let us denote by $\left(2 R T_{0}\right)^{1 / 2} \zeta$ the molecular velocity and introduce the notation $\zeta=|\boldsymbol{\zeta}|$ and $\mu=\zeta_{i} n_{i} / \zeta$, where $-1 \leq \mu \leq 1$ and $0 \leq \zeta<\infty$. Then, the component problems for the Knudsen layer admit a similarity solution of three arguments $(\eta, \mu, \zeta)$ and are reduced to the following two types of boundary-value problems by assuming the hard-sphere molecules and the diffuse reflection boundary condition:

$$
\begin{aligned}
\mu \zeta \frac{\partial \phi_{\alpha}}{\partial \eta} & =-\nu(\zeta) \phi_{\alpha}+\mathcal{C}\left[\phi_{\alpha}\right]-\mathcal{I}_{\alpha}(\eta, \mu, \zeta) \\
\phi_{\alpha} & =-\sigma_{\alpha}^{(0)}-c_{\alpha}^{(0)} \zeta^{2}+g_{\alpha}(\mu, \zeta), \quad(\mu \zeta>0, \eta=0) \\
\phi_{\alpha} & \rightarrow 0, \text { as } \eta \rightarrow \infty
\end{aligned}
$$

and

$$
\begin{aligned}
\mu \zeta \frac{\partial \psi_{\beta}}{\partial \eta} & =-\nu(\zeta) \psi_{\beta}+\mathcal{C}^{S}\left[\psi_{\beta}\right]-\mathcal{I}_{\beta}^{S}(\eta, \mu, \zeta), \\
\psi_{\beta} & =-2 b_{\beta}^{(1)}+g_{\beta}^{S}(\mu, \zeta), \quad(\mu \zeta>0, \eta=0) \\
\psi_{\beta} & \rightarrow 0, \text { as } \eta \rightarrow \infty
\end{aligned}
$$

Here

$$
\nu(\zeta)=\frac{1}{2 \sqrt{2}}\left[\exp \left(-\zeta^{2}\right)+\left(2 \zeta+\frac{1}{\zeta}\right) \int_{0}^{\zeta} \exp \left(-\xi^{2}\right) d \xi\right]
$$

$\mathcal{C}$ is an integral operator acting on a function of $\boldsymbol{\zeta}$ to be defined soon later; and $g_{\alpha}, \mathcal{I}_{\alpha}, g_{\beta}^{S}$, and $\mathcal{I}_{\beta}^{S}$ are given functions. $\mathcal{I}_{\alpha}$ and $\mathcal{I}_{\beta}^{S}$ are supposed to decay fast in $\eta$. The solution $\phi_{\alpha}\left(\right.$ or $\left.\psi_{\beta}\right)(\alpha=1, \ldots, 6 ; \beta=1, \ldots, 8)$ is a function of $\eta, \mu$, and $\zeta$. It is determined together with the constants $\sigma_{\alpha}^{(0)}$ and $c_{\alpha}^{(0)}\left[\right.$ or $\left.b_{\beta}^{(1)}\right]$ for every given $\left(\mathcal{I}_{\alpha}, g_{\alpha}\right)$ or $\left.\left(\mathcal{I}_{\beta}^{S}, g_{\beta}^{S}\right)\right][26]$. The operator $\mathcal{C}$ is defined by

$$
\begin{aligned}
\mathcal{C}[\phi](\boldsymbol{\zeta}) & =\int\left[k_{1}(\boldsymbol{\zeta}, \boldsymbol{\xi})-k_{2}(\boldsymbol{\zeta}, \boldsymbol{\xi})\right] \phi(\boldsymbol{\xi}) d \boldsymbol{\xi} \\
k_{1}(\boldsymbol{\zeta}, \boldsymbol{\xi}) & =\frac{1}{\sqrt{2} \pi|\boldsymbol{\zeta}-\boldsymbol{\xi}|} \exp \left(-|\boldsymbol{\xi}|^{2}+\frac{|\boldsymbol{\xi} \times \boldsymbol{\zeta}|^{2}}{|\boldsymbol{\xi}-\boldsymbol{\zeta}|^{2}}\right), \\
k_{2}(\boldsymbol{\zeta}, \boldsymbol{\xi}) & =\frac{|\boldsymbol{\zeta}-\boldsymbol{\xi}|}{2 \sqrt{2} \pi} \exp \left(-|\boldsymbol{\xi}|^{2}\right) .
\end{aligned}
$$

Thanks to its spherical and axial symmetry, $\mathcal{C}\left[\phi_{\alpha}\right]$ becomes a function of $\mu$ and $\zeta$ (and $\eta$ ) for the function $\phi_{\alpha}(\eta, \mu, \zeta)$. On the other hand, $\mathcal{C}^{S}$ is defined for functions of $\mu$ and $\zeta$ (and $\eta$ ) through $\mathcal{C}$, making use of its axial symmetry:

$$
\zeta_{i} t_{i} \mathcal{C}^{S}\left[\psi_{\beta}\right]=\mathcal{C}\left[\zeta_{i} t_{i} \psi_{\beta}\right]
$$

Once the solutions are obtained, $\Omega_{\alpha}^{(0)}(\eta), \Theta_{\alpha}^{(0)}(\eta), Y_{\beta}^{(1)}(\eta)$ occurring in $(2)$ and $H_{\beta}^{(1)}(\eta)$ in Appendix A are obtained as their moment:

$$
\Omega_{\alpha}^{(0)}(\eta)=\left\langle\phi_{\alpha}\right\rangle, \Theta_{\alpha}^{(0)}(\eta)=\frac{2}{3}\left\langle\left(\zeta^{2}-\frac{3}{2}\right) \phi_{\alpha}\right\rangle,
$$




$$
Y_{\beta}^{(1)}(\eta)=\frac{1}{2}\left\langle\zeta^{2}\left(1-\mu^{2}\right) \psi_{\beta}\right\rangle, H_{\beta}^{(1)}(\eta)=\frac{1}{2}\left\langle\zeta^{2}\left(1-\mu^{2}\right)\left(\zeta^{2}-\frac{5}{2}\right) \psi_{\beta}\right\rangle,
$$

where $\langle f\rangle=\int f(\boldsymbol{\xi}) E(|\boldsymbol{\xi}|) d \boldsymbol{\xi}$ with $E(z)=\pi^{-3 / 2} \exp \left(-z^{2}\right)$.

Our present concern is the case $\alpha=4$ and $\beta=5 \sim 8$, for which $\left(\mathcal{I}_{\alpha}, g_{\alpha}\right)$ and $\left(\mathcal{I}_{\beta}^{S}, g_{\beta}^{S}\right)$ are given as

$$
\begin{aligned}
\mathcal{I}_{4} & =\zeta\left(1-\mu^{2}\right) \frac{\partial \phi_{1}}{\partial \mu}(\eta, \mu, \zeta)-\zeta^{2}\left(1-\mu^{2}\right) \psi_{2}(\eta, \mu, \zeta), \\
g_{4} & =-4 \mu \zeta \int_{0}^{\infty} Y_{2}^{(1)}(z) d z-b_{2}^{(1)} \zeta^{2}\left(1-3 \mu^{2}\right) B(\zeta), \\
\mathcal{I}_{5}^{S} & =\frac{1}{2} \zeta\left(1-\mu^{2}\right) \frac{\partial \psi_{1}}{\partial \mu}(\eta, \mu, \zeta), g_{5}^{S}=-2 D_{1}(\zeta)-\frac{1}{2} \zeta^{2}\left(1-\mu^{2}\right) D_{2}(\zeta), \\
\mathcal{I}_{6}^{S} & =\frac{1}{2} \zeta\left(1-\mu^{2}\right) \frac{\partial \psi_{1}}{\partial \mu}(\eta, \mu, \zeta)-\mu \zeta \psi_{1}(\eta, \mu, \zeta), \\
g_{6}^{S} & =-D_{1}(\zeta)-\frac{1}{2} \zeta^{2}\left(1-3 \mu^{2}\right) D_{2}(\zeta), \\
\mathcal{I}_{7}^{S} & =\frac{1}{2} \zeta\left(1-\mu^{2}\right) \frac{\partial \psi_{2}}{\partial \mu}(\eta, \mu, \zeta)-\mu \zeta \psi_{2}(\eta, \mu, \zeta)+\phi_{1}(\eta, \mu, \zeta), g_{7}^{S}=0, \\
\mathcal{I}_{8}^{S} & =\frac{1}{2} \zeta\left(1-\mu^{2}\right) \frac{\partial \psi_{2}}{\partial \mu}(\eta, \mu, \zeta), g_{8}^{S}=0 .
\end{aligned}
$$

Here $\phi_{1}$ is the solution of the problem (4) with $\mathcal{I}_{1}=0$ and $g_{1}=\mu \zeta A(\zeta)$ (the so-called temperature-jump problem [19]), while $\psi_{1}$ and $\psi_{2}$ are respectively the solution of the problem (5) with $\mathcal{I}_{1}^{S}=0, g_{1}^{S}=\mu \zeta B(\zeta), \mathcal{I}_{2}^{S}=0$, and $g_{2}^{S}=A(\zeta)$ (the so-called shear-slip and thermal-slip [6,27] problems). The pair $\left(b_{2}^{(1)}, Y_{2}^{(1)}\right)$ is the slip coefficient and Knudsen-layer function associated with $\psi_{2}$, which have already been obtained in [20]. Note that all of $\mathcal{I}_{4}$ and $\mathcal{I}_{5}^{S} \sim \mathcal{I}_{8}^{S}$ in (8) indeed decay fast in $\eta$ because of the fast decay of $\phi_{1}, \psi_{1}$, and $\psi_{2}$. The functions $A, B, D_{1}$, and $D_{2}$ of $\zeta$ are familiar solutions of the following integral equations:

$$
\begin{aligned}
& \mathcal{L}\left[\zeta_{i} A(\zeta)\right]=-\zeta_{i}\left(\zeta^{2}-5 / 2\right), \quad \text { subsidiary condition: }\left\langle\zeta^{2} A(\zeta)\right\rangle=0 \\
& \mathcal{L}\left[\zeta_{i j} B(\zeta)\right]=-2 \zeta_{i j}, \\
& \mathcal{L}\left[\left(\zeta_{i} \delta_{j k}+\zeta_{j} \delta_{k i}+\zeta_{k} \delta_{i j}\right) D_{1}(\zeta)+\zeta_{i} \zeta_{j} \zeta_{k} D_{2}(\zeta)\right] \\
& =\gamma_{1}\left(\zeta_{i} \delta_{j k}+\zeta_{j} \delta_{k i}+\zeta_{k} \delta_{i j}\right)-\zeta_{i} \zeta_{j} \zeta_{k} B(\zeta) \\
& \quad \text { subsidiary condition: }\left\langle 5 \zeta^{2} D_{1}(\zeta)+\zeta^{4} D_{2}(\zeta)\right\rangle=0
\end{aligned}
$$

where $\mathcal{L}[f]=-\nu(\zeta) f+\mathcal{C}[f]$ and $\zeta_{i j}=\zeta_{i} \zeta_{j}-(1 / 3) \zeta^{2} \delta_{i j}$.

The essential difficulty which newly appears is that the inhomogeneous term $\mathcal{I}_{\alpha}$ or $\mathcal{I}_{\beta}^{S}$ contains the derivative of the first order Knudsen-layer solution, i.e., $\partial \phi_{1} / \partial \mu, \partial \psi_{1} / \partial \mu$, or $\partial \psi_{2} / \partial \mu$, because $\phi_{1}, \psi_{1}$, and $\psi_{2}$ behave like $\mu \zeta \ln |\mu \zeta|$ for $\mu \zeta \sim-0$ on the boundary and thus their derivatives logarithmically diverge [25]. Our approach to be developed in the sequel follows the method in $[18,25]$ and, in addition, separates the difficult part essentially from the others, thereby dealing with the problem safely numerically. 


\section{Numerical Analysis}

Because (4) and (5) are linear problems, they can be treated by first decomposing the sources $\mathcal{I}_{\alpha}$ (or $\mathcal{I}_{\beta}^{S}$ ) and $g_{\alpha}\left(\right.$ or $g_{\beta}^{S}$ ) as we like and then superposing the individual results. In the present section, we shall concentrate on the cases that all the terms without $\partial \phi_{1} / \partial \mu, \partial \psi_{1} / \partial \mu$, and $\partial \psi_{2} / \partial \mu$ in those sources are absent. Hence, we drop the subscript $\alpha$ from (4) and put $\mathcal{I}=\zeta\left(1-\mu^{2}\right) \partial \phi_{1} / \partial \mu$ and $g=0$, while we put $\beta=A, B$ in (5) with $\mathcal{I}_{A}^{S}=\zeta\left(1-\mu^{2}\right) \partial \psi_{1} / \partial \mu$, $\mathcal{I}_{B}^{S}=\zeta\left(1-\mu^{2}\right) \partial \psi_{2} / \partial \mu, g_{A}^{S}=0$ and $g_{B}^{S}=0$. Note that $\psi_{8}=\frac{1}{2} \psi_{B}$. The remaining parts $\bar{\phi}_{4} \equiv \phi_{4}-\phi, \bar{\psi}_{5,6} \equiv \psi_{5,6}-\frac{1}{2} \psi_{A}$, and $\bar{\psi}_{7} \equiv \psi_{7}-\frac{1}{2} \psi_{B}$ can be handled without difficulty by the method in our previous paper [18], the explanation of which is omitted here.

\subsection{Integral Formulation}

Multiplied by $E(\zeta)$ and integrated with respect to $\eta$, both of the problems (4) and (5) are transformed into

$$
\begin{aligned}
\Phi(\eta, \mu, \zeta)= & G(\mu, \zeta) \exp \left(-\frac{\nu(\zeta) \eta}{\mu \zeta}\right)+\frac{1}{\mu \zeta} \int_{0}^{\eta} C[\Phi](s, \mu, \zeta) \exp \left(\frac{\nu(\zeta)(s-\eta)}{\mu \zeta}\right) d s \\
& +\frac{1}{\mu \zeta} \int_{0}^{\eta} C[\Psi](s, \mu, \zeta) \exp \left(\frac{\nu(\zeta)(s-\eta)}{\mu \zeta}\right) d s, \quad(\mu \zeta>0), \\
\Phi(\eta, \mu, \zeta)= & \frac{1}{\mu \zeta} \int_{\infty}^{\eta} C[\Phi](s, \mu, \zeta) \exp \left(\frac{\nu(\zeta)(s-\eta)}{\mu \zeta}\right) d s \\
& +\frac{1}{\mu \zeta} \int_{\infty}^{\eta} C[\Psi](s, \mu, \zeta) \exp \left(\frac{\nu(\zeta)(s-\eta)}{\mu \zeta}\right) d s, \quad(\mu \zeta<0),
\end{aligned}
$$

with

$$
\begin{aligned}
& \Phi(\eta, \mu, \zeta) \rightarrow 0, \quad \text { as } \eta \rightarrow \infty, \\
& \Psi(\eta, \mu, \zeta)= \begin{cases}-\frac{1}{\mu \zeta} \int_{0}^{\eta} I(s, \mu, \zeta) \exp \left(\frac{\nu(\zeta)(s-\eta)}{\mu \zeta}\right) d s, & (\mu \zeta>0), \\
-\frac{1}{\mu \zeta} \int_{\infty}^{\eta} I(s, \mu, \zeta) \exp \left(\frac{\nu(\zeta)(s-\eta)}{\mu \zeta}\right) d s, & (\mu \zeta<0) .\end{cases}
\end{aligned}
$$

Here, $\Phi, C, I$, and $G$ should be read as $\Phi=\phi E-\Psi, C[f]=\mathcal{C}\left[f E^{-1}\right] E$, $I=\mathcal{I} E$, and $G=\left(-\sigma^{(0)}-c^{(0)} \zeta^{2}\right) E$ for problem (4), while they should be $\operatorname{read}$ as $\Phi=\psi_{\beta} E-\Psi, C[f]=\mathcal{C}^{S}\left[f E^{-1}\right] E, I=\mathcal{I}_{\beta}^{S} E$, and $G=-2 b_{\beta}^{(1)} E$ for problem (5), where $\beta=A, B$. Remember that $I$ decays fast in $\eta$, so does $\Psi$. In the above, the original solution $\phi E, \psi_{A} E$, or $\psi_{B} E$ is split into the given part $\Psi$ and unknown part $\Phi$. The equation for $\Phi$ is none other than the integral form of the inhomogeneous Boltzmann equation with $C[\Psi]$ being its inhomogeneous term. The conditions (4c) and (5c) are reduced to (9c), because $\Psi \rightarrow 0$ as $\eta \rightarrow \infty$. The condition (9c) is required, otherwise the constants $\sigma^{(0)}, c^{(0)}, b_{A}^{(1)}$, and $b_{B}^{(1)}$ are not determined [26]. 
Since $C$ is the integral operator, $C[f]$ is milder than $f$. Nevertheless, because of the singular nature of $I$ considered here, a special attention should be made in the present analysis. In fact, the most difficult part in the present work is the computation of $\Psi$ and $C[\Psi]$, especially their values in approaching the boundary $(\eta \rightarrow 0)$.

Using the facts that $\phi_{1}$ is the solution of (4) with $\mathcal{I}_{1}=0, g_{1}=\mu \zeta A(\zeta)$ and that $\psi_{1}$ and $\psi_{2}$ are the solutions of (5) with $\mathcal{I}_{1}^{S}=0, g_{1}^{S}=\mu \zeta B(\zeta), \mathcal{I}_{2}^{S}=$ $0, g_{2}^{S}=A(\zeta), \Psi$ can be written as

$$
\begin{aligned}
\Psi= & \left(1-\mu^{2}\right) \zeta\left\{-(\frac{\partial h_{0}}{\partial \mu}+\underbrace{\frac{\zeta \nu}{2 \eta}\left|\frac{\eta}{\mu \zeta}\right|^{2} h_{0}}) \frac{\eta}{\mu \zeta} e^{-\frac{\nu \eta}{\mu \zeta}}+\frac{1}{|\mu \zeta|^{2}}\right. \\
& \left.\times \int_{0}^{\eta}\{\underbrace{\zeta\left(1-\frac{\nu}{2}\left|\frac{\eta-s}{\mu \zeta}\right|\right) C[h]}-|\mu \zeta| \frac{\partial C[h]}{\partial \mu}\}\left|\frac{\eta-s}{\mu \zeta}\right| e^{-\nu\left|\frac{\eta-s}{\mu \zeta}\right|} d s\right\},(\mu \zeta>0), \\
\Psi= & -\frac{\left(1-\mu^{2}\right) \zeta}{|\mu \zeta|^{2}} \int_{\eta}^{\infty}\{\underbrace{\left(\zeta\left(1-\frac{\nu}{2}\left|\frac{\eta-s}{\mu \zeta}\right|\right) C[h]\right.}+|\mu \zeta| \frac{\partial C[h]}{\partial \mu}\} \\
& \times\left|\frac{\eta-s}{\mu \zeta}\right| e^{-\nu\left|\frac{\eta-s}{\mu \zeta}\right|} d s,(\mu \zeta<0),
\end{aligned}
$$

where $h$ is $\phi_{1} E, \psi_{1} E$, or $\psi_{2} E$, while $h_{0}$ its value at $\eta=0$; accordingly $C[h]=$ $\mathcal{C}\left[\phi_{1}\right] E, \mathcal{C}^{S}\left[\psi_{1}\right] E$, or $\mathcal{C}^{S}\left[\psi_{2}\right] E$. Note that $C[h]$ is a function of $s, \mu$, and $\zeta$ and that the argument $\zeta$ of $\nu$ and $E$ has been omitted just for conciseness in the above. The parts with the underbrace require a special attention in the computation, which we explain in Sect. 4.2.

\subsection{Singular Properties of $\Psi$}

Let us introduce the following notation:

$$
\begin{aligned}
W[f] & \equiv-\left(1-\mu^{2}\right) \frac{\zeta^{2} \nu}{2 \eta} f(\mu, \zeta)\left|\frac{\eta}{\mu \zeta}\right|^{3} e^{-\frac{\nu \eta}{\mu \zeta}},(\mu \zeta>0), \\
T[f] & \equiv \begin{cases}\frac{\left(1-\mu^{2}\right) \zeta^{2}}{|\mu \zeta|^{2}} \int_{0}^{\eta}\left|\frac{\eta-s}{\mu \zeta}\right|\left(1-\frac{\nu}{2}\left|\frac{\eta-s}{\mu \zeta}\right|\right) f(s, \mu, \zeta) e^{-\nu\left|\frac{\eta-s}{\mu \zeta}\right|} d s, & (\mu \zeta>0), \\
-\frac{\left(1-\mu^{2}\right) \zeta^{2}}{|\mu \zeta|^{2}} \int_{\eta}^{\infty}\left|\frac{\eta-s}{\mu \zeta}\right|\left(1-\frac{\nu}{2}\left|\frac{\eta-s}{\mu \zeta}\right|\right) f(s, \mu, \zeta) e^{-\nu\left|\frac{\eta-s}{\mu \zeta}\right|} d s, & (\mu \zeta<0) .\end{cases}
\end{aligned}
$$

The parts with the underbrace in (10) are then written as $W\left[h_{0}\right]$ and $T[C[h]]$, respectively; the equation (10) is recast as

$$
\begin{aligned}
\Psi= & W\left[h_{0}\right]+T[C[h]]-\left(1-\mu^{2}\right) \zeta\left\{\frac{\partial h_{0}}{\partial \mu} \frac{\eta}{\mu \zeta} e^{-\frac{\nu \eta}{\mu \zeta}}\right. \\
& \left.+\frac{1}{|\mu \zeta|} \int_{0}^{\eta} \frac{\partial C[h]}{\partial \mu}\left|\frac{\eta-s}{\mu \zeta}\right| e^{-\nu\left|\frac{\eta-s}{\mu \zeta}\right|} d s\right\},(\mu \zeta>0),
\end{aligned}
$$




$$
\Psi=T[C[h]]-\frac{\left(1-\mu^{2}\right) \zeta}{|\mu \zeta|} \int_{\eta}^{\infty} \frac{\partial C[h]}{\partial \mu}\left|\frac{\eta-s}{\mu \zeta}\right| e^{-\nu\left|\frac{\eta-s}{\mu \zeta}\right|} d s, \quad(\mu \zeta<0) .
$$

We consider the behavior of $W\left[h_{0}\right]$ and $T[C[h]]$ in three limiting processes: (i) $\eta \rightarrow 0$ with $|\mu \zeta|(>0)$ fixed, (ii) $|\mu \zeta| \rightarrow 0$ with $\eta(>0)$ fixed, and (iii) $\eta,|\mu \zeta| \rightarrow 0$ with $z \equiv\left|\frac{\nu \eta}{\mu \zeta}\right|(>0)$ fixed. Note that $z \rightarrow 0$ in the limit (i), while $z \rightarrow \infty$ in the limit (ii).

As is clear from its form, $W\left[h_{0}\right]$ behaves well in the limits (i) and (ii). It grows, however, in proportion to $\eta^{-1}$ in the limit (iii). Therefore, $W\left[h_{0}\right]$ is singular for $\mu \zeta=+0$ and $\eta=0$.

As to $T[C[h]]$, we need to pay attention to that, even though $C[h]$ behaves well with respect to $\mu$ and $\zeta$, its derivative in $s$ may diverge logarithmically $[18,25]$ as $s \rightarrow 0$. Therefore, as in [25], we put

$$
C[h](s, \mu, \zeta)=a(\mu, \zeta)+b(\mu, \zeta) s \ln s+c(\mu, \zeta) s,
$$

for small $s$ and estimate the behavior of $T[C[h]]$ for $\eta \ll 1$. Then, for $\mu \zeta>0$, we have

$$
\begin{aligned}
\frac{\nu^{3} T[C[h]]}{\left(1-\mu^{2}\right) \zeta^{2}}= & \frac{z^{3}}{2 \eta} e^{-z} a(\mu, \zeta)-\left\{\left(\frac{z^{2}}{2}+z+1\right) e^{-z}[\operatorname{Ei}(z)-\ln z-\gamma]-\frac{z}{2}\right. \\
& +\underbrace{\left[\left(\frac{z^{2}}{2}+z+1\right) e^{-z}-1\right] \ln \eta}-\frac{5}{2}\left(1-e^{-z}\right)+\left(\frac{z^{2}}{2}+2 z\right) e^{-z}\} b(\mu, \zeta) \\
& +\left\{1-\left(\frac{z^{2}}{2}+z+1\right) e^{-z}\right\} c(\mu, \zeta),
\end{aligned}
$$

where $\operatorname{Ei}(z)=$ p.v. $\int_{-\infty}^{z} y^{-1} e^{y} d y,(z>0)$ and $\gamma$ is the Euler constant $(\gamma=$ $0.577216 \ldots)$. Taking into account the properties $\operatorname{Ei}(z)-\gamma-\ln z=O(z)$ for $z \ll 1$ and $e^{-z} \operatorname{Ei}(z)=z^{-1}+O\left(z^{-2}\right)$ for $z \gg 1$, we see that all the terms are finite in the limit (i). In the limit (ii), the part with the underbrace approaches $-\ln \eta$; thus $T[C[h]]$ grows in proportion to $\ln \eta$ as $\eta \rightarrow 0$ at $\mu \zeta=+0$. In the limit (iii), the terms with $a$ and $b$ grow in proportion to $\eta^{-1}$ and to $\ln \eta$, respectively. On the other hand, for $\mu \zeta<0$, we have

$$
\begin{aligned}
\frac{\nu^{3} T[C[h]]}{\left(1-\mu^{2}\right) \zeta^{2}}= & -\frac{w^{3} e^{-w}}{2\left|\eta-\eta_{*}\right|} a(\mu, \zeta)-\{\underbrace{(z-1) e^{z} \mathrm{E}_{1}(z)-\ln \eta}-\frac{z}{2}\left[z e^{z} \mathrm{E}_{1}(z)-1\right] \\
& +\left(\ln \eta_{*}\right) e^{-w}\left[\left(\frac{z^{2}}{2}+\frac{y^{2}}{2}-z+1\right)(1+y)-z y^{2}\right] \\
& +\frac{y}{2}\left[y e^{y} \mathrm{E}_{1}(y)-1\right] e^{-w}-e^{-w}\left[(y-1)(1+w)-\frac{w^{2}}{2}\right] e^{y} \mathrm{E}_{1}(y) \\
& \left.-\frac{5}{2}\left(1-e^{-w}\right)+e^{-w}\left(\frac{w^{2}}{2}+2 w\right)\right\} b(\mu, \zeta) \\
& +\left\{1-\left[1+w+\frac{w^{2}}{2}(1+z)+\frac{w^{3}}{2}\right] e^{-w}\right\} c(\mu, \zeta) \\
& -\left|\frac{\nu}{\mu \zeta}\right|^{2} \int_{\eta_{*}}^{\infty} \nu\left|\frac{\eta-s}{\mu \zeta}\right|\left(1-\frac{\nu}{2}\left|\frac{\eta-s}{\mu \zeta}\right|\right) C[h](s, \mu, \zeta) e^{-\nu\left|\frac{\eta-s}{\mu \zeta}\right|} d s
\end{aligned}
$$


where $\mathrm{E}_{1}(z)=\int_{z}^{\infty} y^{-1} e^{-y} d y,(z>0), w \equiv \nu\left|\frac{\eta_{*}-\eta}{\mu \zeta}\right|, y \equiv z+w=\left|\frac{\nu \eta_{*}}{\mu \zeta}\right|$, and $\eta_{*}$ is a fixed positive constant such that $3 \eta<\eta_{*} \ll 1$. In the above three limiting processes, $w$ and $y$ behave respectively as (i) $w \rightarrow y$ with $y(>0)$ fixed, (ii) $w, y \rightarrow \infty$, and (iii) $w, y \rightarrow \infty$; and in all cases $\frac{z}{w}$ and $\frac{y}{w}$ are finite. The integral on the last line is seen to be bounded by

$$
\frac{w}{\eta_{*}-\eta}\left(\frac{1}{2} w^{2}+2 w+2\right) e^{-w} \max _{\eta \geq \eta_{*}} C[h],
$$

and always remains finite in those limits. However, because of the properties $e^{x} \mathrm{E}_{1}(x)+\gamma+\ln x=O(x \ln x)$ for $x \ll 1$ and $e^{x} \mathrm{E}_{1}(x)=x^{-1}+O\left(x^{-2}\right)$ for $x \gg 1$, it is seen that the part with the underbrace approaches $\gamma+\ln \left|\frac{\nu}{\mu \zeta}\right|$ in the limit (i) and grows in proportion to $\ln \eta$ in the limit (iii). The limit (i) implies that $T[C[h]]$ grows with the rate $\ln |\mu \zeta|$ as $\mu \zeta \rightarrow-0$ on the boundary $\eta=0$. In the limit (ii), it approaches $1-\ln \eta$; thus $T[C[h]]$ grows in proportion to $\ln \eta$ as $\eta \rightarrow 0$ at $\mu \zeta=-0$.

To summarize, $\Psi$ exhibits the singular behavior and may diverge for $\eta, \mu \zeta \sim$ 0 . To be specific, when $\mu \zeta<0$, it diverges with the rate $\ln \eta$ as $\eta \rightarrow 0$ with $\left|\frac{\nu \eta}{\mu \zeta}\right|$ fixed and with the rate $\ln |\mu \zeta|$ as $\mu \zeta \rightarrow 0$ for $\eta=0$. When $\mu \zeta>0$, it diverges with the rate $\eta^{-1}$ as $\eta \rightarrow 0$ with $\left|\frac{\nu \eta}{\mu \zeta}\right|$ fixed due to both $W\left[h_{0}\right]$ and $T[C[h]]$, especially the contribution from the part $a(\mu, \zeta)$ of $C[h]$ for the latter. When $\mu \zeta= \pm 0$, it grows with the rate $\ln \eta$ as $\eta \rightarrow 0$.

\subsection{Influence of the Singularity of $\Psi$ on Its Moments and $C[\Psi]$}

Due to the behavior studied in Sect. 4.2, $C[\Psi]$ and the moments of $\Psi$ on the boundary should be considered carefully. We define them as the values in the limit $\eta \rightarrow 0$. Then, a special attention should be made to the singularity in the limit (iii); the order of the limit $\eta \rightarrow 0$ and the integration with respect to $\mu$ and $\zeta$ is not allowed to change naively. We have obtained the following identities, by which the moments of $\Psi$ for $\eta=0$ can be computed appropriately numerically:

$$
\begin{gathered}
\lim _{\eta \rightarrow 0} \int_{\mu \zeta>0} \mathcal{P}(\mu, \zeta) W\left[h_{0}\right] d \zeta=2 \pi \lim _{\eta \rightarrow 0} \int_{0}^{\infty} \int_{0}^{1} \zeta^{2} \mathcal{P}(\mu, \zeta) W\left[h_{0}\right] d \mu d \zeta \\
=-2 \pi \lim _{\eta \rightarrow 0} \int_{0}^{\infty} \int_{0}^{1}\left(1-\mu^{2}\right) \frac{\zeta^{4}}{2 \nu^{2}} \frac{1}{\eta}\left|\frac{\nu \eta}{\mu \zeta}\right|^{3} e^{-\frac{\nu \eta}{\mu \zeta}} \mathcal{P}(\mu, \zeta) h_{0}(\mu, \zeta) d \mu d \zeta \\
=-\pi \int_{0}^{\infty} \frac{\zeta^{3}}{\nu} \mathcal{P}(0, \zeta) h_{0}(0, \zeta) d \zeta=-\pi \int_{0}^{\infty} \frac{\zeta^{3}}{\nu} \mathcal{P}(0, \zeta) h(0,+0, \zeta) d \zeta, \quad \\
\lim _{\eta \rightarrow 0} \int_{\mu \zeta<0} \mathcal{P}(\mu, \zeta) T[C[h]] d \zeta=2 \pi \lim _{\epsilon \downarrow 0} \int_{0}^{\infty} \int_{-1}^{-\epsilon} \mathcal{P}(\mu, \zeta) \lim _{\eta \rightarrow 0} \zeta^{2} T[C[h]] d \mu d \zeta,
\end{gathered}
$$

$$
\lim _{\eta \rightarrow 0} \int_{\mu \zeta>0} \mathcal{P}(\mu, \zeta) T[C[h]] d \zeta=2 \pi \lim _{\eta \rightarrow 0} \int_{0}^{\infty} \int_{0}^{1} \zeta^{2} \mathcal{P}(\mu, \zeta) T[C[h]] d \mu d \zeta
$$




$$
\begin{aligned}
& =\pi \lim _{\eta \rightarrow 0} \frac{1}{\eta} \int_{0}^{\infty} \int_{0}^{1}\left|\frac{\nu \eta}{\mu \zeta}\right|^{3} e^{-\left|\frac{\nu \eta}{\mu \zeta}\right|} \mathcal{P}(\mu, \zeta) a(\mu, \zeta) \frac{\left(1-\mu^{2}\right) \zeta^{4}}{\nu^{3}} d \mu d \zeta \\
& =\pi \int_{0}^{\infty} \frac{\zeta^{3}}{\nu^{2}} \mathcal{P}(0, \zeta) a(0, \zeta) d \zeta=\pi \int_{0}^{\infty} \frac{\zeta^{3}}{\nu^{2}} \mathcal{P}(0, \zeta) C[h](0,0, \zeta) d \zeta
\end{aligned}
$$

where $\mathcal{P}$ is a regular function of its arguments [see Appendix B for the derivation of (16)]. Therefore, we have

$$
\begin{array}{r}
\lim _{\eta \rightarrow 0} \int \mathcal{P}(\mu, \zeta) \Psi(\eta, \mu, \zeta) d \zeta=2 \pi \lim _{\epsilon \downarrow 0} \int_{0}^{\infty} \int_{-1}^{-\epsilon} \mathcal{P}(\mu, \zeta) \zeta^{2} \Psi(0, \mu, \zeta) d \mu d \zeta \\
+\pi \int_{0}^{\infty} \frac{\zeta^{3}}{\nu^{2}} \mathcal{P}(0, \zeta)\{C[h](0,0, \zeta)-\nu h(0,+0, \zeta)\} d \zeta
\end{array}
$$

As for $C[\Psi]$, it can be written in the form

$$
C[\Psi](\eta, \mu, \zeta)=\int_{0}^{\infty} \int_{-1}^{1} \int_{0}^{2 \pi} \xi^{2} K(M, \xi, \theta ; \mu, \zeta) \Psi(\eta, M, \xi) d \theta d M d \xi
$$

where $\theta$ is the angle between the projections of $\boldsymbol{\xi}$ and $\boldsymbol{\zeta}$ onto the plane normal to their common polar direction $(M=\mu=1)$. The key difference of the above $K$ from $\mathcal{P}$ is that it has a singularity of $|\boldsymbol{\xi}-\boldsymbol{\zeta}|^{-1}$. Fortunately, however, the singularity can be removed by transformation of integral variables, so that we obtain the identity similar to (17):

$$
\begin{gathered}
C[\Psi](0, \mu, \zeta) \equiv \lim _{\eta \rightarrow 0} \int_{0}^{\infty} \int_{-1}^{1} \int_{0}^{2 \pi} \xi^{2} K(M, \xi, \theta ; \mu, \zeta) \Psi(\eta, M, \xi) d \theta d M d \xi \\
=\lim _{\epsilon \downarrow 0} \int_{0}^{\infty} \int_{-1}^{-\epsilon} \int_{0}^{2 \pi} K(M, \xi, \theta ; \mu, \zeta) \xi^{2} \Psi(0, M, \xi) d \theta d M d \xi \\
+\int_{0}^{\infty} \int_{0}^{2 \pi} \frac{\xi^{3}}{2 \nu^{2}} K(0, \xi, \theta ; \mu, \zeta)\{C[h](0,0, \xi)-\nu h(0,+0, \xi)\} d \theta d \xi
\end{gathered}
$$

The required calculation becomes lengthy and we omit it in the present paper.

\subsection{Discretization and Some Details of Numerical Method}

Thanks to the factor $E$, both of $\Phi$ and $\Psi$ are expected to decay rapidly in $\zeta$, as well as in $\eta$. We thus truncate the $\zeta$ - and $\eta$-spaces at $\zeta=Z$ and $\eta=d$ for properly chosen positive constants $Z$ and $d$ and restrict the computation in the region $-1 \leq \mu \leq 1,0 \leq \zeta \leq Z$, and $0 \leq \eta \leq d$. The chosen values of $Z$ and $d$ are justified a posteriori from the results. Non-uniform discretization is made for each variables. The grid in molecular velocity space $(\mu, \zeta)$ is arranged two-fold: one is the grid for capturing the milder function $C[\Phi]$ (and $C[\Psi]$ ), while the other is that for capturing $\Phi$ (and $\Psi$ ) to compute $C[\Phi]$ (and $C[\Psi]$ ) enough accurately. The latter grid should be finer than the former. In the case of the standard grid, i.e., (S1,M1) in [18], we set $Z=5.0$ and $d=44.46$, and 
arrange 251 grid points in $\eta$ space; the two-fold grid in $\mu$ - $\zeta$ space consists of $257 \times 141$ and $449 \times 161$ points. $^{1}$ More details are found in [18].

For the spatial coordinate $\eta$, we arrange $2 N_{\eta}+1$ grid points, say $\eta^{(i)}$ $\left(i=0,1, \ldots, 2 N_{\eta}\right)$, in the region $0 \leq \eta \leq d$ :

$$
0=\eta^{(0)}<\eta^{(1)}<\cdots<\eta^{\left(2 N_{\eta}\right)}=d .
$$

As the primary grid for the molecular velocity space, we arrange $\left(4 N_{\mu}+1\right) \times$ $\left(2 N_{\zeta}+1\right)$ points, say $\left(\mu^{(j)}, \zeta^{(k)}\right)\left(j=-2 N_{\mu}, \ldots, 2 N_{\mu} ; k=0, \ldots, 2 N_{\zeta}\right)$ in the region $-1 \leq \mu \leq 1$ and $0 \leq \zeta \leq Z$ :

$$
\begin{aligned}
& 0=\mu^{(0)}<\mu^{(1)}<\cdots<\mu^{\left(2 N_{\mu}-1\right)}<\mu^{\left(2 N_{\mu}\right)}=1, \\
& \mu^{(-j)}=-\mu^{(j)},\left(1 \leq j \leq 2 N_{\mu}\right), \\
& 0=\zeta^{(0)}<\zeta^{(1)}<\cdots<\zeta^{\left(2 N_{\zeta}\right)}=Z
\end{aligned}
$$

while, as the secondary grid, we arrange $\left(4 N_{M}+1\right) \times\left(2 N_{\xi}+1\right)$ points in $\mu-\zeta$ space, say $\left(M^{(l)}, \xi^{(m)}\right)\left(l=-2 N_{M}, \ldots, 2 N_{M} ; m=0, \ldots, 2 N_{\xi}\right)$ :

$$
\begin{aligned}
& 0=M^{(0)}<M^{(1)}<\cdots<M^{\left(2 N_{M}-1\right)}<M^{\left(2 N_{M}\right)}=1, \\
& M^{(-l)}=-M^{(l)},\left(1 \leq l \leq 2 N_{M}\right) \\
& 0=\xi^{(0)}<\xi^{(1)}<\cdots<\xi^{\left(2 N_{\xi}\right)}=Z .
\end{aligned}
$$

Note that $N_{M}>N_{\mu}$ and $N_{\xi}>N_{\zeta}$. Once the reliable data of $C[\Psi]$ is obtained, the solution method for (9) is the same as that in [18]. Hence, we explain below how to obtain $\Psi$ and $C[\Psi]$.

As is obvious from (9), in order to compute $\Phi$, the information of $C[\Psi]$ on all grid points $\left(\eta^{(i)}, \mu^{(j)}, \zeta^{(k)}\right)$ is required. For $\eta^{(i)}$ with $i \neq 0, C[\Psi]$ is computed in the same way as the computation of $C[\Phi]$, which is based on the piecewise quadratic interpolation of $\Psi$ in the molecular velocity space (see also [18]). For $\eta^{(0)}$, however, $C[\Psi]$ is computed in accordance with (18) in Sect. 4.3, where $C[h](0,0, \xi)$ is interpolated piecewise quadratically in $\xi$ in the last integral. As to the first integral, the region of integration with respect to $M$ is truncated from $-1<M<-\epsilon$ with $\epsilon \rightarrow+0$ to $-1<M<M^{(-2)}$ for the integration of $T[C[h]]$ in $\Psi$ [see (12)]; no truncation is made for the integration of the reminder $\Psi-T[C[h]]$. In both integrations, $T[C[h]]$ or $\Psi-T[C[h]]$ is interpolated piecewise quadratically both in $M$ and $\xi$. The truncation causes the error of $O\left(M^{(-2)} \ln \left|M^{(-2)}\right|\right)$, which can be controlled to be small enough by a proper arrangement of the secondary grid. In computing the contribution from $\Psi$ to the Knudsen-layer functions at $\eta=0,(17)$ is treated in the same way.

Finally, we turn to the computation of $\Psi$. As is clear from the previous paragraph, we have already handled with the divergence of $\Psi$ at $\eta=0$ and do not have to worry about it. When computing $\Psi$ by (12), we use the following

\footnotetext{
1 Using the notation appearing soon below, $N_{\eta}=125, N_{\mu}=64, N_{\zeta}=70, N_{M}=112$, and $N_{\xi}=80$.
} 


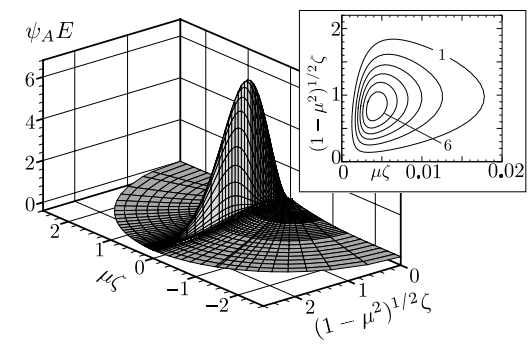

(a) $\eta=0.015$

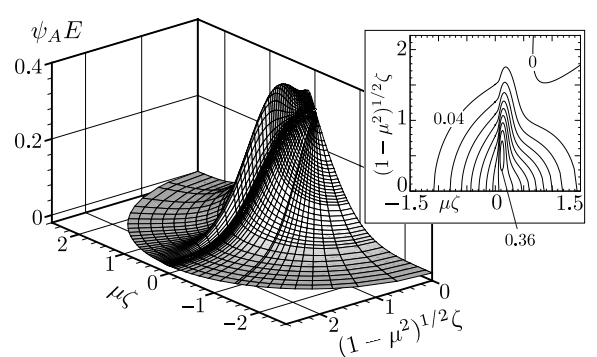

(b) $\eta=0.58$

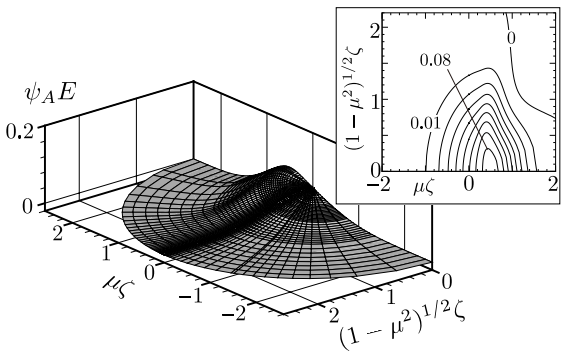

(c) $\eta=3.0$

Fig. $1 \psi_{A} E$ and its contour plots at three spatial points. (a) $\eta=0.015$, (b) $\eta=0.58$, and (c) $\eta=3.0$. In the contour plots, the curves are drawn with the intervals 1 in (a), 0.04 in (b), and 0.01 in (c).

piecewise interpolation with respect to $s$ for the part $T[C[h]]$ in the range $0<s \leq \eta^{(2 \sigma)}$, where $\sigma$ is a certain small natural number, [see (13)]:

$$
\begin{aligned}
& C[h]\left(s, \mu^{(j)}, \zeta^{(k)}\right)=a\left(\mu^{(j)}, \zeta^{(k)}\right)+b\left(\mu^{(j)}, \zeta^{(k)}\right) s \ln s+c\left(\mu^{(j)}, \zeta^{(k)}\right) s, \\
& \text { for } \quad \eta^{(2 i)}<s \leq \eta^{(2 i+2)}, i=0,1, \ldots, \sigma-1 .
\end{aligned}
$$

Here the coefficients $a, b$, and $c$ are determined in such a way that $C[h](s, \cdot, \cdot)$ takes the values $C[h]\left(\eta^{(2 i)}, \cdot, \cdot\right), C[h]\left(\eta^{(2 i+1)}, \cdot, \cdot\right)$, and $C[h]\left(\eta^{(2 i+2)}, \cdot, \cdot\right)$ at $s=$ $\eta^{(2 i)}, \eta^{(2 i+1)}$, and $\eta^{(2 i+2)}$. As to the remaining part $\Psi-T[C[h]]$ in that range and $\Psi$ in the remaining range, we use a piecewise quadratic interpolation with respect to $s$, as in [18]. The value of $\eta^{(2 \sigma)}$ is about 0.1 in all of our computations.

\section{Numerical Results}

The results in the present section are obtained by the standard grid (S1,M1) in [18], unless otherwise stated. The measure of accuracy of the present computations is given in Appendix C. Further data, in addition to those covering the results in $[10,18]$, are available from the authors. 


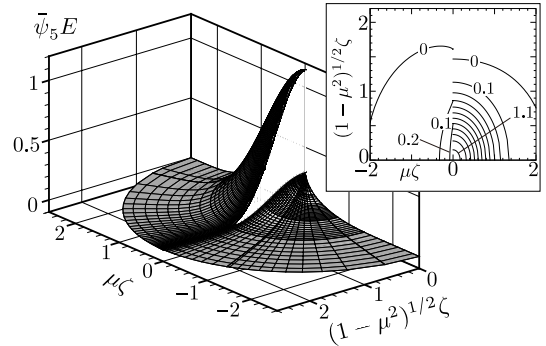

(a) $\eta=0$

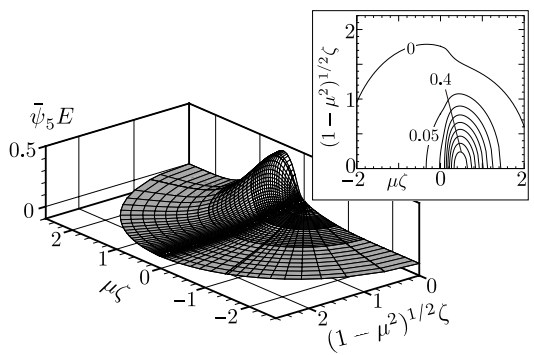

(c) $\eta=0.58$

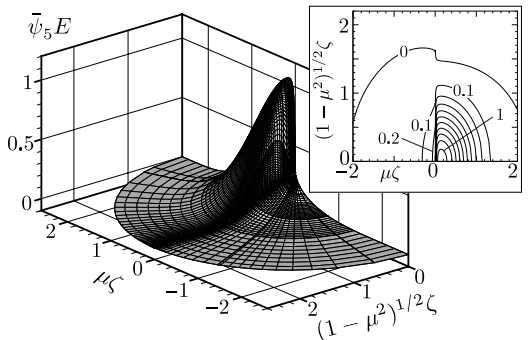

(b) $\eta=0.015$

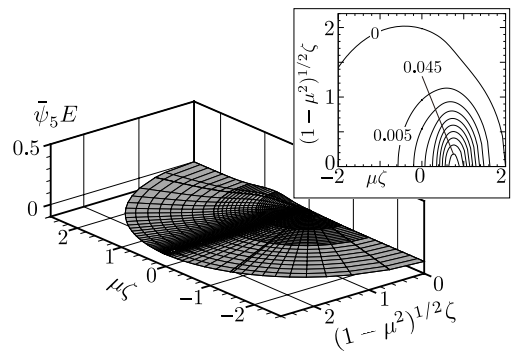

(d) $\eta=3.0$

Fig. $2 \bar{\psi}_{5} E$ and its contour plots at four spatial points. (a) $\eta=0$, (b) $\eta=0.015$, (c) $\eta=$ 0.58 , and (d) $\eta=3.0$. In the contour plots the curves are drawn with the intervals 0.1 in (a) and (b), 0.05 in (c), and 0.005 in (d). The white vertical surface at $\mu \zeta=0$ in (a) shows the discontinuity.

\subsection{Velocity Distribution Functions}

As an illustrative example, we show $\psi_{A} E$ at three spatial points $\eta=0.015$, $0.58,3.0$ in Fig. 1 and $\bar{\psi}_{5} E$ at four spatial points $\eta=0,0.015,0.58,3.0$ in Fig. 2. It should be reminded that $\psi_{5}=\frac{1}{2} \psi_{A}+\bar{\psi}_{5}$ (see the first paragraph of Sect. 4) and that $\psi_{A}$ contains a diverging part at $\eta \sim 0$ and $\mu \zeta \sim 0$, while $\bar{\psi}_{5}$ does not. Due to this fact, the scale of the vertical axis is changed largely in Fig. 1, while it is not changed in Fig. 2. In Fig. $1, \psi_{A} E$ on the boundary $\eta=0$ is not drawn by the same fact.

In Fig. 2, the discontinuity is clearly observed along the line $\mu \zeta=0$ on the boundary $\eta=0$, which is the line corresponding to the molecular velocity tangential to the boundary (see Fig. 2a). The discontinuity vanishes immediately away from the boundary with keeping the other part almost unchanged. Accordingly, a continuous but steep variation appears in the molecular velocity space near the boundary (see Fig. $2 \mathrm{~b}$ ). These features are also observed in $\bar{\phi}_{4}, \bar{\psi}_{6}, \bar{\psi}_{7}$, and the solutions obtained in [18].

In order to see the singular behavior of the velocity distribution functions more closely, we show in Fig. $3 \eta \phi, \eta \psi_{A}$, and $\eta \psi_{B}$ for small $\eta$ as a function of $\mu / \eta(>0)$. It is seen that the profiles of $\eta \phi, \eta \psi_{A}$, and $\eta \psi_{B}$ tend to converge individually as $\eta$ decreases. This confirms that $\phi, \psi_{A}$, and $\psi_{B}$ diverge in proportion to $\eta^{-1}$ for $\mu \zeta>0$ in the limit (iii) of Sect. 4.2 , due to the corre- 


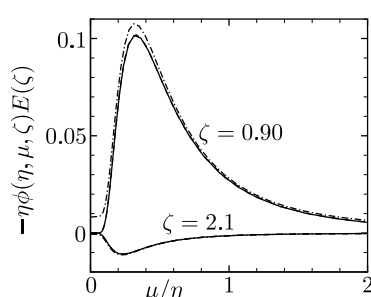

(a)

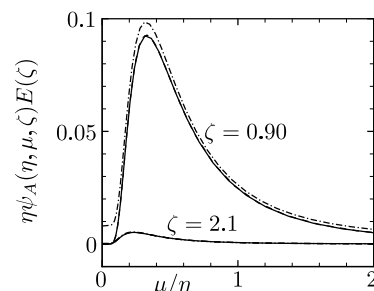

(b)

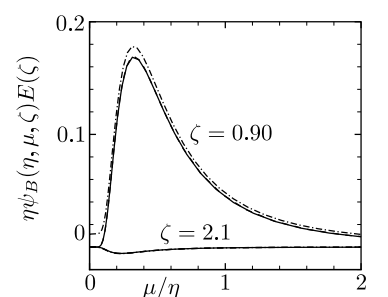

(c)

Fig. 3 Behavior of $\phi, \psi_{A}$, and $\psi_{B}$ for small $\eta$. (a) $\eta \phi E$ vs $\mu / \eta$, (b) $\eta \psi_{A} E$ vs $\mu / \eta$, and (c) $\eta \psi_{B} E$ vs $\mu / \eta$. Three different lines are drawn for each value of $\zeta$ in each panel: the solid line represents the result for $\eta=7.3 \times 10^{-7}$, the dashed line that for $\eta=1.4 \times 10^{-4}$, and the dash-dotted line that for $\eta=1.5 \times 10^{-2}$. The dashed line almost agrees with the solid line and is invisible for $\zeta=0.90$. Three lines almost agree with one another and the difference is invisible for $\zeta=2.1$.

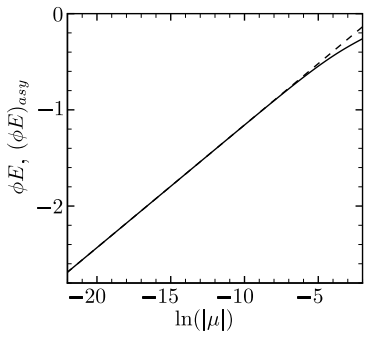

(a)

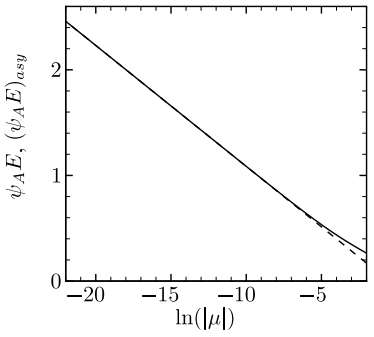

(b)

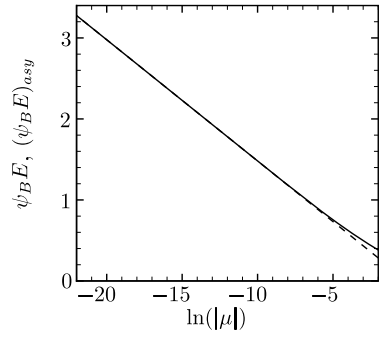

(c)

Fig. 4 Behavior of $\phi E, \psi_{A} E$, and $\psi_{B} E$ for $\mu \lesssim 0$ on the boundary $\eta=0$, with $\zeta$ being fixed $(\zeta=0.90)$. (a) $\phi E$, (b) $\psi_{A} E$, and (c) $\psi_{B} \widetilde{E}$. The solid line represents the numerical solution of $\phi E, \psi_{A} E$, and $\psi_{B} E$, while the dashed line $(\phi E)_{a s y},\left(\psi_{A} E\right)_{a s y}$, and $\left(\psi_{B} E\right)_{a s y}$ predicted theoretically [see (20)].

sponding property of the part $\Psi$. Another close observation can be made for the limit (i) of Sect. 4.2 for $\mu \zeta<0$. From (15), we see that for $|\mu| \ll 1$

$$
T[C[h]](\eta=0, \mu<0, \zeta)=\frac{\zeta^{2}}{\nu^{3}}\left[\left(\frac{5}{2}-\gamma-\ln \frac{\nu}{|\mu \zeta|}\right) b(0, \zeta)+c(0, \zeta)\right]+O(\mu \ln |\mu|),
$$

which in turn leads to the following asymptotic behavior:

$$
\begin{aligned}
\varphi(\eta=0, \mu<0, \zeta) \rightarrow & (\varphi)_{a s y} \equiv \frac{\zeta^{2}}{\nu^{3}}\left[\left(\frac{5}{2}-\gamma-\ln \frac{\nu}{|\mu \zeta|}\right) b(0, \zeta)+c(0, \zeta)\right] \\
& -\frac{\zeta}{\nu^{2}} \frac{\partial C[h]}{\partial \mu}(0,0, \zeta)+\frac{1}{\nu} C[\varphi](0,0, \zeta), \quad \text { as } \mu \nearrow 0,
\end{aligned}
$$

where $\varphi=\phi E, \psi_{A} E, \psi_{B} E$ for $h=\phi_{1} E, \psi_{1} E, \psi_{2} E$. Note that $b$ and $c$ depend on $h$. We show in Fig. 4 the numerical data of $\varphi(\eta=0, \mu<0, \zeta)$ for small $|\mu|$ by the solid line. It is clearly observed that, as $\mu \nearrow 0$, the numerical solution of $\phi E, \psi_{A} E$, or $\psi_{B} E$ on the boundary approaches $(\phi E)_{a s y},\left(\psi_{A} E\right)_{a s y}$, or $\left(\psi_{B} E\right)_{a s y}$, i.e., the dashed line. This again shows that our numerical method 
Table 1 Slip/jump coefficients. The data in [10] obtained by the use of the symmetry relation [23] are also shown for comparisons.

\begin{tabular}{ccllll}
\hline & $c_{4}^{(0)}$ & \multicolumn{1}{c}{$b_{5}^{(1)}$} & \multicolumn{1}{c}{$b_{6}^{(1)}$} & $b_{7}^{(1)}$ & \multicolumn{1}{c}{$b_{8}^{(1)}$} \\
\hline Present results & 4.6180 & -0.66012 & 0.24381 & 0.44728 & -0.23353 \\
Symmetry relation [10] & 4.6181 & -0.6601 & 0.2438 & 0.4472 & -0.2336 \\
\hline
\end{tabular}

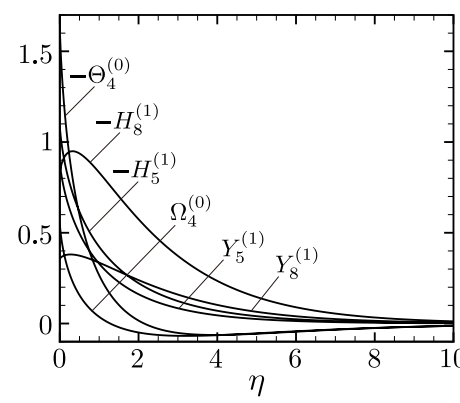

(a)

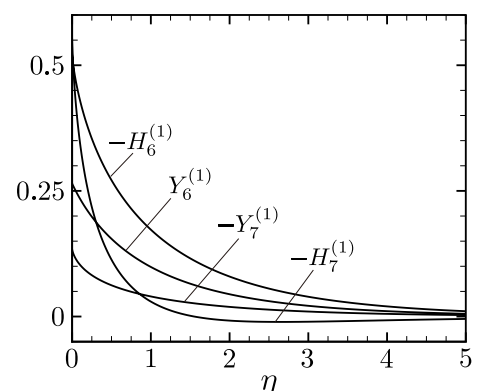

(b)

Fig. 5 Knudsen-layer functions. (a) Knudsen-layer functions for $\phi_{4}, \psi_{5}$, and $\psi_{8}$, (b) those for $\psi_{6}$ and $\psi_{7}$.

works consistently enough to capture the logarithmic divergence of the solution in $|\mu|$ for $\mu \nearrow 0$ on the boundary discussed in Sect. 4.2.

\subsection{Slip/Jump Coefficients and Knudsen-Layer Functions}

Slip/jump coefficients are shown in Table 1. The data in [10], which are obtained from the information about the first-order Knudsen layer by the theory of symmetry relation [23], are also shown for comparisons. Excellent agreement ensures the consistency of the present results to the theory.

The Knudsen-layer functions $\Omega_{4}^{(0)}, \Theta_{4}^{(0)}, Y$ 's, and $H$ 's are shown in Fig. 5 and Table 2 [see Appendix A for $H$ 's and (2) for the others]. Corresponding to the behavior of the velocity distribution functions in $\eta$, they decay fast as $\eta \rightarrow \infty$, mostly monotonically except for $\Omega_{4}^{(0)}, \Theta_{4}^{(0)}$ and $H_{7}^{(1)}$ (see Table 2). From the table, the $90 \%$ thickness of the Knudsen layer is seen to be about $4 \sim 5$ for $\Omega_{4}^{(0)}$, about $1 \sim 1.5$ for $\Theta_{4}^{(0)}$, about $2.5 \sim 3$ for $Y_{5}^{(1)}, H_{5}^{(1)}, Y_{6}^{(1)}$, $H_{6}^{(1)}$, and $Y_{7}^{(1)}$, about $0.5 \sim 1$ for $H_{7}^{(1)}$, and about $6 \sim 7$ for $Y_{8}^{(1)}$ and $H_{8}^{(1)}$, measured in $\eta$.

\section{Concluding Remarks}

In the present paper, we have investigated the curvature effects in the generalized slip-flow theory [4-6], which occur at the second order of the Knudsen number expansion, and thereby complete the series of data required for its 
Table 2 Knudsen-layer functions.

\begin{tabular}{|c|c|c|c|c|c|c|c|c|c|c|}
\hline$\eta$ & $\Omega_{4}^{(0)}$ & $-\Theta_{4}^{(0)}$ & $Y_{5}^{(1)}$ & $-H_{5}^{(1)}$ & $Y_{6}^{(1)}$ & $-H_{6}^{(1)}$ & $-Y_{7}^{(1)}$ & $-H_{7}^{(1)}$ & $Y_{8}^{(1)}$ & $-H_{8}^{(1)}$ \\
\hline 0.00000 & 0.55644 & 1.64676 & 0.89334 & 1.09051 & 0.26440 & 0.52114 & 0.13408 & 0.55360 & 0.35551 & 0.82712 \\
\hline 0.02348 & & & 4419 & 3017 & & 19034 & & & & 55993 \\
\hline & 41 & 38089 & 80432 & & & & & & & \\
\hline & 9296 & 123232 & 75158 & 91763 & 23478 & 13116 & 19 & 0.34719 & 0.37368 & 99 \\
\hline & & & & 6124 & 22146 & 0119 & & & & 92837 \\
\hline 0.19 & 1052 & 1520 & 67030 & 82045 & 21118 & 37946 & & 0.25677 & .37929 & .93838 \\
\hline & 55885 & & & & & & & 0477 & & \\
\hline & & & & 53 & & & & & & 915 \\
\hline & & & 18 & & & & & & & \\
\hline & & & & & & & & & & 4624 \\
\hline & & & & & & & & 170 & & 2453 \\
\hline & & & & & & & & & & \\
\hline & & & & & & & & & & \\
\hline & & & & & & & & & & \\
\hline & & & & & & & & & & 169 \\
\hline & & & & & & & & & & \\
\hline & & & & & & & & & & \\
\hline & & & & & & & & & & \\
\hline & & & & & & & & & & \\
\hline & & & & & & & & & & \\
\hline & & & & & & & & & & \\
\hline & & & & & & & & & & \\
\hline & & & & & & & & & & \\
\hline & & & & & & & & & & \\
\hline & & & & & & & & & & 753 \\
\hline & & & & & & & & & & \\
\hline & & & & & & & & & & 708 \\
\hline & & & & & & & & & & \\
\hline & & & & & & & & & & \\
\hline & & & & & & & & & & 175 \\
\hline & & & & & & & & & & \\
\hline & & & & & & & & & & \\
\hline & & & & & & & & & & \\
\hline & & & & & & & & & & \\
\hline 35.11181 & 0.00000 & 0.00000 & 0.00000 & 0.00000 & 0.00000 & 0.00000 & 0.00000 & 0.00000 & 0.00000 & 0.00 \\
\hline
\end{tabular}

application to practical problems. This also completes our recent studies on the time-dependent slip-flow theory $[10,18]$ for a slightly rarefied gas. In the course of analyses, we have also clarified that the curvature effects induce the local divergence singularity of the velocity distribution function, even though it is integrable to make macroscopic quantities well defined. We have obtained the precise information on the singularity and thereby have established a numerical method that handles it appropriately.

The occurrence of the local divergence above can be understood physically from the viewpoint of the boundary geometry. In the generalized slip-flow theory, the study of the Knudsen-layer structure is reduced to a series of spatially half-space problems. It implies that the boundary is approximated as if the boundary were flat at each stage of the analysis. As a result, the solution at the first order of the Knudsen number is forced to be discontinuous on the boundary in the tangential direction of molecular velocity, resulting in the divergence singularity at the second order through the inhomogeneous term. Actually, however, if the curvature exists, the behavior of that discontinuity dramatically changes. For instance, when the boundary is the surface of a convex body, that discontinuity exists but propagates into the gas region in the tangential directions of the boundary. Then, the implicit assumption of continuous solution in the analysis breaks down. The region with this break- 
down, which is thinner by one order of the Knudsen number in its thickness, is called the S-layer [6], which was discovered in [28] and was recognized later in [29] as the manifestation of the discontinuity propagation. The estimate of corrections to the generalized slip-flow theory in the S-layer can be found in $[6,29]$. When the boundary is a surface of concave body, the velocity distribution function becomes continuous even on the boundary. These facts near the convex/concave boundary illustrate that the flattened boundary treatment in the asymptotic analysis does not capture the very local structure of the velocity distribution function, when the curvature exists. Improvement of the local analytical drawback could be an interesting subject in the mathematical physics.

Acknowledgements The present work is supported in part by KAKENHI from JSPS (Nos. 23360083 and 13J01011). The authors thank Professor Kazuo Aoki, Kyoto University, for his interest and encouragement.

\section{A Stress and Heat Flow}

The stress tensor and heat-flow vector are also familiar fluid-dynamic quantities that become necessary, most typically, in computing the momentum and energy exchange with the body surface. Denoting the former by $p_{0}\left(\delta_{i j}+P_{i j}\right)$ and the latter by $p_{0}\left(2 R T_{0}\right)^{1 / 2} Q_{i}$, their Hilbert part $h_{\mathrm{H}}=h_{\mathrm{H} 0}+h_{\mathrm{H} 1} \varepsilon+\cdots$ and Knudsen-layer correction $h_{\mathrm{K}}=h_{\mathrm{K} 0}+h_{\mathrm{K} 1} \varepsilon+\cdots \quad(h=$ $\left.P_{i j}, Q_{i}\right)$ up to the second order in $\varepsilon$ are summarized as follows:

$$
\begin{aligned}
& P_{i j \mathrm{H} m}=P_{\mathrm{H} m} \delta_{i j}-\gamma_{1} \frac{\overline{\partial u_{i \mathrm{H} m-1}}}{\partial x_{j}}+\frac{1}{2} \gamma_{3} \frac{\overline{\partial^{2} \tau_{\mathrm{H} m-2}}}{\partial x_{i} \partial x_{j}},(m=0,1,2), \\
& Q_{i \mathrm{H} m}=-\frac{5}{4} \gamma_{2} \frac{\partial \tau_{\mathrm{H} m-1}}{\partial x_{i}}+\frac{1}{2} \gamma_{3} \frac{\partial^{2} u_{i \mathrm{H} m-2}}{\partial x_{j}^{2}},(m=0,1,2),
\end{aligned}
$$

and

$$
\begin{aligned}
P_{i j \mathrm{~K} m}= & \frac{3}{2} \frac{\partial \tau_{\mathrm{H} m-1}}{\partial x_{k}} n_{k}\left(\delta_{i j}-n_{i} n_{j}\right)\left[\Omega_{1}^{(0)}(\eta)+\Theta_{1}^{(0)}(\eta)\right],(m=0,1), \\
P_{i j \mathrm{~K} 2} n_{i} n_{j}= & -3 \bar{\kappa} \frac{\partial \tau_{\mathrm{H} 0}}{\partial x_{i}} n_{i} \int_{\eta}^{\infty}\left[\Omega_{1}^{(0)}(z)+\Theta_{1}^{(0)}(z)\right] d z, \\
P_{i j \mathrm{~K} 2} n_{i} t_{j}= & \frac{3}{2}\left(\frac{\partial^{2} \tau_{\mathrm{H} 0}}{\partial x_{i} \partial x_{j}} n_{i} t_{j}+\kappa_{i j} t_{j} \frac{\partial \tau_{\mathrm{H} 0}}{\partial x_{i}}\right) \int_{\eta}^{\infty}\left[\Omega_{1}^{(0)}(z)+\Theta_{1}^{(0)}(z)\right] d z, \\
Q_{i \mathrm{~K} m} t_{i}= & \frac{\partial u_{i \mathrm{H} m-1}}{\partial x_{j}} n_{i} t_{j} H_{1}^{(1)}(\eta)+\frac{\partial \tau_{\mathrm{H} m-1}}{\partial x_{i}} t_{i} H_{2}^{(1)}(\eta) \\
& +\frac{\partial^{2} \tau_{\mathrm{H} m-2}}{\partial x_{i} \partial x_{j}} n_{i} t_{j} H_{3}^{(1)}(\eta)+\frac{\partial}{\partial x_{i}} \frac{\frac{\partial u_{j \mathrm{H} m-2}}{\partial x_{k}}}{\frac{\partial u_{j} \mathrm{H} m-2}{\partial x_{k}}} n_{j} t_{k} t_{i} H_{4}^{(1)}(\eta)(\eta) \\
& +\bar{\kappa} \frac{\partial u_{i \mathrm{H} m-2}}{\partial x_{j}} n_{i} t_{j} H_{5}^{(1)}(\eta)+\kappa_{i j} \frac{\partial)}{\partial x_{k}} \\
+ & \kappa_{i j} t_{j} \frac{\partial \tau_{\mathrm{H} m-2}}{\partial x_{i}} H_{7}^{(1)}(\eta)+\bar{\kappa} \frac{\partial \tau_{\mathrm{H} m-2}}{\partial x_{i}} t_{i} H_{8}^{(1)}(\eta), \quad(m=0,1,2),
\end{aligned}
$$




$$
\begin{aligned}
Q_{i \mathrm{~K} m} n_{i}= & {\left[\frac{\partial^{2} \tau_{\mathrm{H} m-2}}{\partial x_{i} \partial x_{j}}\left(\delta_{i j}-n_{i} n_{j}\right)-2 \bar{\kappa} \frac{\partial \tau_{\mathrm{H} m-2}}{\partial x_{i}} n_{i}\right] \int_{\eta}^{\infty} H_{2}^{(1)}(z) d z } \\
& -\frac{1}{2} \frac{\partial}{\partial x_{i}} \frac{\overline{\partial u_{j \mathrm{H} m-2}}}{\partial x_{k}} n_{i} n_{j} n_{k} \int_{\eta}^{\infty} H_{1}^{(1)}(z) d z, \quad(m=0,1,2) .
\end{aligned}
$$

Here the quantities with the subscript $\mathrm{H}$ in (22) denote their value on the boundary. The functions $H_{1}^{(1)} \sim H_{4}^{(1)}$ have already been obtained in [18, 20,21]. The present work newly provides the data of $H_{5}^{(1)} \sim H_{8}^{(1)}$, which are included in Table 2 and Fig. 5.

\section{B Sketch of Derivation of (16)}

The non-trivial equality in (16a) is

$$
\lim _{\eta \rightarrow 0} \int_{0}^{\infty} \int_{0}^{1}\left(1-\mu^{2}\right) \frac{\zeta^{4}}{\nu^{2}} \frac{1}{\eta}\left|\frac{\nu \eta}{\mu \zeta}\right|^{3} e^{-\frac{\nu \eta}{\mu \zeta}} \mathcal{P}(\mu, \zeta) h_{0}(\mu, \zeta) d \mu d \zeta=\int_{0}^{\infty} \frac{\zeta^{3}}{\nu} \mathcal{P}(0, \zeta) h_{0}(0, \zeta) d \zeta .
$$

Once (23) is proved, the still non-trivial equality in (16c) is

$$
\begin{aligned}
2 \lim _{\eta \rightarrow 0} \int_{0}^{\infty} \int_{0}^{1} \zeta^{2} \mathcal{P}(\mu, \zeta) T[C[h]] d \mu d \zeta \\
=\lim _{\eta \rightarrow 0} \frac{1}{\eta} \int_{0}^{\infty} \int_{0}^{1}\left|\frac{\nu \eta}{\mu \zeta}\right|^{3} e^{-\left|\frac{\nu \eta}{\mu \zeta}\right|} \mathcal{P}(\mu, \zeta) a(\mu, \zeta) \frac{\left(1-\mu^{2}\right) \zeta^{4}}{\nu^{3}} d \mu d \zeta
\end{aligned}
$$

We show below the outline of the proof for (23), (24), and (16b).

Proof of (23) We first split the region of integration with respect to $\mu$ into $(0, \delta)$ and $(\delta, 1)$, where $0<\delta<1$ is a constant. Then for the second part, we see that

$$
\begin{aligned}
\mid \int_{0}^{\infty} \int_{\delta}^{1} & \frac{\zeta^{4}}{\nu^{2}} \frac{1}{\eta}\left|\frac{\nu \eta}{\mu \zeta}\right|^{3} e^{-\frac{\nu \eta}{\mu \zeta}}\left\{\left(1-\mu^{2}\right) \mathcal{P}(\mu, \zeta) h_{0}(\mu, \zeta)-\mathcal{P}(0, \zeta) h_{0}(0, \zeta)\right\} d \mu d \zeta \mid \\
\leq & \int_{0}^{\infty} \int_{\delta}^{1} \frac{\zeta^{4}}{\nu^{2}} \frac{1}{\eta}\left|\frac{\nu \eta}{\delta \zeta}\right|^{3} e^{-\frac{\nu \eta}{\zeta}} \max _{\mu}\left(\left|\mathcal{P}(\mu, \zeta) h_{0}(\mu, \zeta)\right|+\left|\mathcal{P}(0, \zeta) h_{0}(0, \zeta)\right|\right) d \mu d \zeta \\
& \leq \delta^{-3} \int_{0}^{\infty} \eta \zeta^{2}\left|\frac{\nu \eta}{\zeta}\right| e^{-\frac{\nu \eta}{\zeta}} \max _{\mu}\left(\left|\mathcal{P}(\mu, \zeta) h_{0}(\mu, \zeta)\right|+\left|\mathcal{P}(0, \zeta) h_{0}(0, \zeta)\right|\right) d \zeta \\
& \leq \delta^{-3} \eta \int_{0}^{\infty} \zeta^{2} \max _{\mu}\left(\left|\mathcal{P}(\mu, \zeta) h_{0}(\mu, \zeta)\right|+\left|\mathcal{P}(0, \zeta) h_{0}(0, \zeta)\right|\right) d \zeta \rightarrow 0, \quad \text { as } \eta \rightarrow 0
\end{aligned}
$$

On the other hand, by applying to $\mathcal{P}$ and $h_{0}$ the mean-value theorem with respect to $\mu$, we have

$$
\begin{aligned}
& \left.\left|\int_{0}^{\infty} \int_{0}^{\delta} \frac{\zeta^{4}}{\nu^{2}} \frac{1}{\eta}\right| \frac{\nu \eta}{\mu \zeta}\right|^{3} e^{-\frac{\nu \eta}{\mu \zeta}}\left\{\left(1-\mu^{2}\right) \mathcal{P}(\mu, \zeta) h_{0}(\mu, \zeta)-\mathcal{P}(0, \zeta) h_{0}(0, \zeta)\right\} d \mu d \zeta \mid \\
& \leq \int_{0}^{\infty} \int_{0}^{\delta} \frac{\zeta^{4}}{\nu^{2}} \frac{1}{\eta}\left|\frac{\nu \eta}{\mu \zeta}\right|^{3} e^{-\frac{\nu \eta}{\mu \zeta}}\left(\left|\mathcal{P}^{\prime} h_{0}(0, \zeta)\right| \mu+\left|\mathcal{P}(0, \zeta) h_{0}^{\prime}\right| \mu+\left|\mathcal{P} h_{0}\right| \mu^{2}+\left|\mathcal{P}^{\prime} h_{0}^{\prime}\right| \mu^{2}\right) d \mu d \zeta \\
& \leq \int_{0}^{\infty} \int_{0}^{\delta} \frac{\zeta^{3}}{\nu}\left|\frac{\nu \eta}{\mu \zeta}\right|^{2} e^{-\frac{\nu \eta}{\mu \zeta}} \max _{\mu}\left(\left|\mathcal{P}^{\prime} h_{0}(0, \zeta)\right|+\left|\mathcal{P}(0, \zeta) h_{0}^{\prime}\right|\right) d \mu d \zeta \\
& +\eta \int_{0}^{\infty} \int_{0}^{\delta} \zeta^{2}\left|\frac{\nu \eta}{\mu \zeta}\right| e^{-\frac{\nu \eta}{\mu \zeta}} \max _{\mu}\left(\left|\mathcal{P} h_{0}\right|+\left|\mathcal{P}^{\prime} h_{0}^{\prime}\right|\right) d \mu d \zeta \\
& \leq \delta \int_{0}^{\infty} \frac{\zeta^{3}}{\nu} \max _{\mu}\left(\left|\mathcal{P}^{\prime} h_{0}(0, \zeta)\right|+\left|\mathcal{P}(0, \zeta) h_{0}^{\prime}\right|\right) d \zeta+\eta \delta \int_{0}^{\infty} \zeta^{2} \max _{\mu}\left(\left|\mathcal{P} h_{0}\right|+\left|\mathcal{P}^{\prime} h_{0}^{\prime}\right|\right) d \zeta
\end{aligned}
$$




$$
\rightarrow \delta \int_{0}^{\infty} \frac{\zeta^{3}}{\nu} \max _{\mu}\left(\left|\mathcal{P}^{\prime} h_{0}(0, \zeta)\right|+\left|\mathcal{P}(0, \zeta) h_{0}^{\prime}\right|\right) d \zeta, \quad \text { as } \eta \rightarrow 0,
$$

where $\mathcal{P}^{\prime}\left(\right.$ or $\left.h_{0}^{\prime}\right)$ is the partial derivative of $\mathcal{P}$ (or $h_{0}$ ) with respect to $\mu$ at $\mu=\mu_{P}\left(\right.$ or $\mu_{h}$ ), and $\mu_{P}\left(\right.$ or $\left.\mu_{h}\right)$ is a certain value in the interval $[0, \mu]$ that depends on $\mu$ and $\zeta$. Therefore, we have

$$
\begin{aligned}
\lim _{\eta \rightarrow 0} \int_{0}^{\infty} \int_{0}^{1}\left(1-\mu^{2}\right) \frac{\zeta^{4}}{\nu^{2}} \frac{1}{\eta}\left|\frac{\nu \eta}{\mu \zeta}\right|^{3} e^{-\frac{\nu \eta}{\mu \zeta}} \mathcal{P}(\mu, \zeta) h_{0}(\mu, \zeta) d \mu d \zeta \\
=\lim _{\eta \rightarrow 0} \int_{0}^{\infty} \int_{0}^{1} \frac{\zeta^{4}}{\nu^{2}} \frac{1}{\eta}\left|\frac{\nu \eta}{\mu \zeta}\right|^{3} e^{-\frac{\nu \eta}{\mu \zeta} \mathcal{P}(0, \zeta) h_{0}(0, \zeta) d \mu d \zeta+O(\delta)} \\
=\lim _{\eta \rightarrow 0} \int_{0}^{\infty} \frac{\zeta^{3}}{\nu}\left(1+\frac{\nu \eta}{\zeta}\right) e^{-\frac{\nu \eta}{\zeta}} \mathcal{P}(0, \zeta) h_{0}(0, \zeta) d \zeta+O(\delta) \\
=\lim _{\eta \rightarrow 0} \int_{0}^{\infty} \frac{\zeta^{3}}{\nu} e^{-\frac{\nu \eta}{\zeta}} \mathcal{P}(0, \zeta) h_{0}(0, \zeta) d \zeta+O(\delta)
\end{aligned}
$$

Now, in the most right-hand side of (25), we can change the order of the limit and integration, because

$$
\begin{aligned}
\left|\int_{0}^{\infty} \frac{\zeta^{3}}{\nu}\left(1-e^{-\frac{\nu \eta}{\zeta}}\right) \mathcal{P}(0, \zeta) h_{0}(0, \zeta) d \zeta\right| & \leq \int_{0}^{\infty} \frac{\zeta}{\nu}\left(1-e^{-\frac{\nu \eta}{\zeta}}\right) \zeta^{2}\left|\mathcal{P}(0, \zeta) h_{0}(0, \zeta)\right| d \zeta \\
& \leq \eta \int_{0}^{\infty} \zeta^{2}\left|\mathcal{P}(0, \zeta) h_{0}(0, \zeta)\right| d \zeta \rightarrow 0, \quad \text { as } \eta \rightarrow 0
\end{aligned}
$$

Here, we have used the fact that $0 \leq x^{-1}\left(1-e^{-x \eta}\right) \leq \eta$ for $x \geq 0$. Therefore, we finally arrive at

$\lim _{\eta \rightarrow 0} \int_{0}^{\infty} \int_{0}^{1}\left(1-\mu^{2}\right) \frac{\zeta^{4}}{\nu^{2}} \frac{1}{\eta}\left|\frac{\nu \eta}{\mu \zeta}\right|^{3} e^{-\frac{\nu \eta}{\mu \zeta}} \mathcal{P}(\mu, \zeta) h_{0}(\mu, \zeta) d \mu d \zeta=\int_{0}^{\infty} \frac{\zeta^{3}}{\nu} \mathcal{P}(0, \zeta) h_{0}(0, \zeta) d \zeta+O(\delta)$.

In the course of estimates, $\delta$ is a positive constant, arbitrary as far as smaller than unity; thus it can be made small as we wish, which proves (23).

Proof of (24) We use the expression (13) of $C[h]$ for small $\eta$ and its consequence (14) for $\mu \zeta>0$, namely

$$
\frac{\nu^{3} T[C[h]]}{\left(1-\mu^{2}\right) \zeta^{2}}=\frac{1}{\eta} h_{1}(z) a(\mu, \zeta)+h_{2}(z) b(\mu, \zeta) \ln \eta+h_{3}(z) b(\mu, \zeta)+h_{2}(z) c(\mu, \zeta),
$$

where

$$
\begin{aligned}
& h_{1}(z)=\frac{1}{2} z^{3} e^{-z}, \quad h_{2}(z)=1-\left(\frac{1}{2} z^{2}+z+1\right) e^{-z} \\
& h_{3}(z)=-\left(\frac{1}{2} z^{2}+z+1\right) e^{-z}[\operatorname{Ei}(z)-\ln z-\gamma]+\frac{1}{2} z+\frac{5}{2}\left(1-e^{-z}\right)-\left(\frac{1}{2} z^{2}+2 z\right) e^{-z}
\end{aligned}
$$

and $h_{1} \sim h_{3}$ are $O\left(z^{3}\right)$ for $z \ll 1$ and $O(1)$ for $z \gg 1$ (remember that $z=\frac{\nu \eta}{\mu \zeta}$ ). Then,

$$
\begin{aligned}
2 \int_{0}^{\infty} \int_{0}^{1} \zeta^{2} \mathcal{P}(\mu, \zeta) T[C[h]] d & \mu d \zeta=2 \int_{0}^{\infty} \int_{0}^{1} \frac{\zeta^{4}}{\nu^{3}}\left(1-\mu^{2}\right) \mathcal{P}(\mu, \zeta)\left[\frac{1}{\eta} h_{1}(z) a(\mu, \zeta)\right. \\
& \left.+h_{2}(z) b(\mu, \zeta) \ln \eta+h_{3}(z) b(\mu, \zeta)+h_{2}(z) c(\mu, \zeta)\right] d \mu d \zeta .
\end{aligned}
$$

For $f(z)$ that follows the same estimate for both $z \ll 1$ and $z \gg 1$ as $h_{1} \sim h_{3}$ and for $Q(\mu, \zeta)$ that is regular and decays rapidly in $\zeta$, we have

$$
\begin{aligned}
\mid \int_{0}^{\infty} & \int_{0}^{1} \frac{\zeta^{4}}{\nu^{3}}\left(1-\mu^{2}\right) f(z) Q(\mu, \zeta) d \mu d \zeta\left|\leq \int_{0}^{\infty} \frac{\zeta^{4}}{\nu^{3}} \max _{\mu}\right| Q\left|\int_{0}^{1}\right| f(z) \mid d \mu d \zeta \\
& \leq \eta \int_{0}^{\infty} \frac{\zeta^{3}}{\nu^{2}} \max _{\mu}|Q| \int_{\frac{\nu \eta}{\zeta}}^{\infty} z^{-2}|f(z)| d z d \zeta \leq \eta \int_{0}^{\infty} \frac{\zeta^{3}}{\nu^{2}} \max _{\mu}|Q| d \zeta \int_{0}^{\infty} z^{-2}|f(z)| d z .
\end{aligned}
$$


Therefore, taking the limit $\eta \rightarrow 0$, all the terms, except the first, on the right-hand side of (26) are seen to vanish. We are left with

$\lim _{\eta \rightarrow 0} 2 \int_{0}^{\infty} \int_{0}^{1} \zeta^{2} \mathcal{P}(\mu, \zeta) T[C[h]] d \mu d \zeta=2 \lim _{\eta \rightarrow 0} \frac{1}{\eta} \int_{0}^{\infty} \int_{0}^{1} \frac{\zeta^{4}}{\nu^{3}}\left(1-\mu^{2}\right) \mathcal{P}(\mu, \zeta) h_{1}(z) a(\mu, \zeta) d \mu d \zeta$

which is none other than (24).

Proof of (16b) We use again the expression (13) of $C[h]$ for small $\eta$ and its consequence (15) for $\mu \zeta<0$. We first rewrite (15) as

$$
\begin{aligned}
T[C[h]]=[ & f_{1}\left(w, \eta, \eta_{*}\right) a(\mu, \zeta)+f_{2}(z, \eta) b(\mu, \zeta)+f_{3}\left(w, z, y, \eta_{*}\right) b(\mu, \zeta) \\
& \left.+f_{4}(w, z) c(\mu, \zeta)+f_{5}\left(\mu, \zeta, \eta, \eta_{*}\right)\right] \frac{\left(1-\mu^{2}\right) \zeta^{2}}{\nu^{3}}, \\
f_{1}= & -\frac{w^{3} e^{-w}}{2\left|\eta-\eta_{*}\right|}, \\
f_{2}= & \ln \eta-(z-1) e^{z} \mathrm{E}_{1}(z)+\frac{z}{2}\left[z e^{z} \mathrm{E}_{1}(z)-1\right], \\
f_{3}= & -\left(\ln \eta_{*}\right) e^{-w}\left[\left(\frac{z^{2}}{2}+\frac{y^{2}}{2}-z+1\right)(1+y)-z y^{2}\right]-\frac{y}{2}\left[y e^{y} \mathrm{E}_{1}(y)-1\right] e^{-w} \\
& +e^{-w}\left[(y-1)(1+w)-\frac{w^{2}}{2}\right] e^{y} \mathrm{E}_{1}(y)+\frac{5}{2}\left(1-e^{-w}\right)-e^{-w}\left(\frac{w^{2}}{2}+2 w\right), \\
f_{4}=1 & -\left(1+w+\frac{w^{2}}{2}(1+z)+\frac{w^{3}}{2}\right) e^{-w}, \\
f_{5}= & -\left|\frac{\nu}{\mu \zeta}\right|^{2} \int_{\eta_{*}}^{\infty} \nu\left|\frac{\eta-s}{\mu \zeta}\right|\left(1-\frac{\nu}{2}\left|\frac{\eta-s}{\mu \zeta}\right|\right) C[h](s, \mu, \zeta) e^{-\nu\left|\frac{\eta-s}{\mu \zeta}\right|} d s,
\end{aligned}
$$

and consider the limit (i) in Sect. 4.2 (remember that $y=\left|\frac{\nu \eta_{*}}{\mu \zeta}\right|$ and $w=y-z$ ). Then, in this limit we have

$$
\begin{aligned}
T[C[h]] \rightarrow\left[f_{1}\left(y, 0, \eta_{*}\right) a(\mu, \zeta)\right. & +f_{2}(0,0) b(\mu, \zeta)+f_{3}\left(y, 0, y, \eta_{*}\right) b(\mu, \zeta) \\
& \left.+f_{4}(y, 0) c(\mu, \zeta)+f_{5}\left(\mu, \zeta, 0, \eta_{*}\right)\right] \frac{\left(1-\mu^{2}\right) \zeta^{2}}{\nu^{3}} \equiv T[C[h]]_{0} .
\end{aligned}
$$

Here a special attention should be paid to the limit for $f_{2}$. It yields $f_{2}(0,0)=-\gamma-\ln \nu+$ $\ln |\mu \zeta|$. The limits for the others are straightforward. As a result, $\int_{\mu \zeta<0} \mathcal{P}(\mu, \zeta) T[C[h]]_{0} d \zeta$ converges, because the integrand diverges only at the rate $\ln |\mu|$. Thanks to the regularity in $\zeta$, the integral is identical to $2 \pi \lim _{\epsilon \downarrow 0} \int_{0}^{\infty} \int_{-1}^{-\epsilon} \mathcal{P}(\mu, \zeta) T[C[h]]_{0} \zeta^{2} d \mu d \zeta$, so that all we have to do is just to show that

$$
\left|\int_{\mu \zeta<0} \mathcal{P}(\mu, \zeta)\left(T[C[h]]-T[C[h]]_{0}\right) d \zeta\right| \rightarrow 0, \quad \text { as } \eta \rightarrow 0 .
$$

To prove this, we estimate the differences occurring in $T[C[h]]-T[C[h]]_{0}$. Firstly,

$$
\begin{aligned}
\Delta f_{1} & \equiv|a|\left|f_{1}\left(w, \eta, \eta_{*}\right)-f_{1}\left(y, 0, \eta_{*}\right)\right|=\frac{y^{3} e^{-y}}{2 \eta_{*}}\left|1-\left(1-\frac{\eta}{\eta_{*}}\right)^{2} e^{\frac{\eta}{\eta_{*}} y}\right||a| \\
& \leq \frac{y^{3} e^{-y}}{2 \eta_{*}}\left|1-e^{\frac{\eta}{\eta_{*}} y}+\frac{\eta}{\eta_{*}}\left(2-\frac{\eta}{\eta_{*}}\right) e^{\frac{\eta}{\eta_{*}} y}\right||a| \leq\left[C_{1}\left(e^{\frac{\eta}{\eta_{*}} y}-1\right)+C_{2} \frac{\eta}{\eta_{*}} e^{\frac{\eta}{\eta_{*}} y}\right]|a| e^{-y / 2},
\end{aligned}
$$


$\Delta f_{3} \equiv|b|\left|f_{3}\left(w, z, y, \eta_{*}\right)-f_{3}\left(y, 0, y, \eta_{*}\right)\right|$

$=|b| \mid\left(\ln \eta_{*}\right) e^{-y}\left\{\left(\frac{y^{2}}{2}+1\right)(1+y)-e^{\frac{\eta}{\eta_{*}} y}\left[\left(\frac{y^{2}}{2} \frac{\eta^{2}}{\eta_{*}^{2}}+\frac{y^{2}}{2}-\frac{\eta}{\eta_{*}} y+1\right)(1+y)-\frac{\eta}{\eta_{*}} y^{3}\right]\right\}$

$+\left(\frac{5}{2}-\frac{y}{2}\right) e^{-y}\left(1-e^{\frac{\eta}{\eta_{*}} y}\right)+\mathrm{E}_{1}(y)\left\{1+\left[(y-1)\left(1+\left|1-\frac{\eta}{\eta_{*}}\right| y\right)\right.\right.$

$\left.\left.-\frac{y^{2}}{2}\left|1-\frac{\eta}{\eta_{*}}\right|^{2}-\frac{y^{2}}{2}\right] e^{\frac{\eta}{\eta_{*}} y}\right\}-\frac{y}{2} e^{-y}\left[e^{\frac{\eta}{\eta_{*}} y}\left|1-\frac{\eta}{\eta_{*}}\right|\left(\left|1-\frac{\eta}{\eta_{*}}\right| y+4\right)-(y+4)\right] \mid$

$\leq|b|\left\{\left|\ln \eta_{*}\right|(1+y)^{3}\left(e^{\frac{\eta}{\eta_{*}} y}-1+\frac{2 \eta}{\eta_{*}} y e^{\frac{\eta}{\eta_{*}} y}\right) e^{-y}+(3+y)\left(e^{\frac{\eta}{\eta_{*}} y}-1\right) e^{-y}\right.$

$\left.+\left[e^{\frac{\eta}{\eta_{*}} y}-1+\frac{\eta}{\eta_{*}} y(1+y) e^{\frac{\eta}{\eta_{*}} y}\right] \mathrm{E}_{1}(y)+\frac{y}{2}(y+4)\left(e^{\frac{\eta}{\eta_{*}} y}-1+\frac{2 \eta}{\eta_{*}} e^{\frac{\eta}{\eta_{*}} y}\right) e^{-y}\right\}$

$\leq|b|\left[C_{1}\left(e^{\frac{\eta}{\eta_{*}} y}-1\right)+C_{2} \frac{\eta}{\eta_{*}} e^{\frac{\eta}{\eta_{*}} y}\right] e^{-y / 2}$,

$\Delta f_{4} \equiv|c|\left|f_{4}(w, z)-f_{4}(y, 0)\right|=|c| e^{-y} \mid y\left\{1-\frac{1}{2} y\left[y-2+\frac{\eta}{\eta_{*}}(1-2 y)+\frac{\eta^{2}}{\eta_{*}^{2}} y\right]\right.$

$\left.+\frac{1}{2} y^{2}\left(3-3 \frac{\eta}{\eta_{*}}+\frac{\eta^{2}}{\eta_{*}^{2}}\right)\right\} \frac{\eta}{\eta_{*}} e^{\frac{\eta}{\eta_{*}} y}+\left(1+y+\frac{1}{2} y^{2}+\frac{1}{2} y^{3}\right)\left(1-e^{\frac{\eta}{\eta_{*}} y}\right) \mid$

$\leq|c|(1+y)^{2}\left[6 y \frac{\eta}{\eta_{*}} e^{\frac{\eta}{\eta_{*}} y}+(1+y)\left(e^{\frac{\eta}{\eta_{*}} y}-1\right)\right] e^{-y}$

$\leq|c|\left[C_{1}\left(e^{\frac{\eta}{\eta_{*}} y}-1\right)+C_{2} \frac{\eta}{\eta_{*}} e^{\frac{\eta}{\eta_{*}} y}\right] e^{-y / 2}$,

$\Delta f_{5} \equiv\left|f_{5}\left(\mu, \zeta, \eta, \eta_{*}\right)-f_{5}\left(\mu, \zeta, 0, \eta_{*}\right)\right|$

$=\left|\frac{\nu}{\mu \zeta}\right|^{3}\left|\int_{\eta_{*}}^{\infty}\left\{(s-\eta)\left(1-\frac{\nu}{2} \frac{s-\eta}{|\mu \zeta|}\right) e^{\frac{\nu \eta}{|\mu \zeta|}}-s\left(1-\frac{\nu}{2} \frac{s}{|\mu \zeta|}\right)\right\} e^{-\frac{\nu s}{|\mu \zeta|}} C[h](s, \mu, \zeta) d s\right|$

$\leq\left|\frac{\nu}{\mu \zeta}\right|^{3} \int_{\eta_{*}}^{\infty}\left|s\left(1-\frac{\nu}{2} \frac{s}{|\mu \zeta|}\right)\left(e^{\frac{\nu \eta}{\mu \zeta \mid}}-1\right)-\eta\left(1-\frac{\nu}{2} \frac{2 s-\eta}{|\mu \zeta|}\right) e^{\frac{\nu \eta}{|\mu \zeta|}}\right| e^{-\frac{\nu s}{|\mu \zeta|}} d s \max _{s \geq \eta_{*}}|C[h]|$

$\leq\left|\frac{\nu}{\mu \zeta}\right|^{3} \int_{\eta_{*}}^{\infty}\left\{s\left(1+\frac{\nu}{2} \frac{s}{|\mu \zeta|}\right)\left(e^{\frac{\nu \eta}{|\mu \zeta|}}-1\right)+\eta\left(1+\frac{\nu s}{|\mu \zeta|}\right) e^{\frac{\nu \eta}{|\mu \zeta|}}\right\} e^{-\frac{\nu s}{|\mu \zeta|}} d s \max _{s \geq \eta_{*}}|C[h]|$

$=\frac{y}{\eta_{*}}\left\{\frac{1}{2}(2+y)^{2}\left(e^{\frac{\eta}{\eta_{*}} y}-1\right)+\frac{\eta}{\eta_{*}} y(2+y) e^{\frac{\eta}{\eta_{*}} y}\right\} e^{-y} \max _{s \geq \eta_{*}}|C[h]|$

$\leq\left[C_{1}\left(e^{\frac{\eta}{\eta_{*}} y}-1\right)+C_{2} \frac{\eta}{\eta_{*}} e^{\frac{\eta}{\eta_{*}} y}\right] e^{-y / 2} \max _{s \geq \eta_{*}}|C[h]|$,

where $C_{1}$ and $C_{2}$ are positive constants, common to $\Delta f_{1}$ and $\Delta f_{3} \sim \Delta f_{5}$, and the arguments of $a(\mu, \zeta), b(\mu, \zeta)$, and $c(\mu, \zeta)$ are omitted for conciseness. In the above estimates, we have used that $z=\left(\eta / \eta_{*}\right) y$ and $0<\eta / \eta_{*}<1 / 3(<1)$. Therefore, for $i=1,3 \sim 5$, we have

$$
\begin{aligned}
& \left|\int_{\mu \zeta<0} \mathcal{P}(\mu, \zeta) \Delta f_{i} \frac{\left(1-\mu^{2}\right) \zeta^{2}}{\nu^{3}} d \zeta\right| \leq 2 \pi \int_{\mu \zeta<0}|\mathcal{P}(\mu, \zeta)| \Delta f_{i} \frac{\zeta^{4}}{\nu^{3}} d \mu d \zeta \\
\leq & 2 \pi \int_{\mu \zeta<0} \tilde{P}(\mu, \zeta)\left(C_{1}\left|e^{\frac{\eta}{\eta_{*}} y}-1\right|+C_{2} \frac{\eta}{\eta_{*}} e^{\frac{\eta}{\eta_{*}} y}\right) e^{-y / 2} \frac{\zeta^{4}}{\nu^{3}} d \mu d \zeta \\
= & 2 \pi \eta_{*} \int_{0}^{\infty} \max _{\mu}|\tilde{P}| \frac{\zeta^{3}}{\nu^{2}} \int_{\frac{\eta_{*} \nu}{\zeta}}^{\infty}\left(C_{1}\left|e^{\frac{\eta}{\eta_{*}} y}-1\right|+C_{2} \frac{\eta}{\eta_{*}} e^{\frac{\eta}{\eta_{*}} y}\right) y^{-2} e^{-y / 2} d y d \zeta \\
\leq & 2 \pi \eta_{*} \int_{0}^{\infty} \max _{\mu}|\tilde{P}| \frac{\zeta^{5}}{\nu^{4}} \frac{1}{\eta_{*}^{2}} \frac{2 C_{1}\left(e^{\frac{\eta \nu}{\zeta}}-1\right)+2 \frac{\eta}{\eta_{*}}\left(C_{2} e^{\frac{\eta \nu}{\zeta}}+2 C_{1}\right)}{1-2 \frac{\eta}{\eta_{*}}} e^{-\frac{\eta_{*} \nu}{2 \zeta}} d \zeta \\
\leq & \frac{12 \pi}{\eta_{*}} \int_{0}^{\infty} \max _{\mu}|\tilde{P}| \frac{\zeta^{5}}{\nu^{4}}\left[C_{1}\left(e^{\frac{\eta \nu}{\zeta}}-1\right) e^{-\frac{\eta_{*} \nu}{2 \zeta}}+\frac{\eta}{\eta_{*}}\left(C_{2}+2 C_{1}\right)\right] d \zeta
\end{aligned}
$$




$$
\begin{aligned}
= & \frac{12 \pi}{\eta_{*}}\left\{\int_{0}^{\sqrt{\eta}}+\int_{\sqrt{\eta}}^{\infty}\right\} \max _{\mu}|\tilde{P}| \frac{\zeta^{5}}{\nu^{4}} C_{1}\left(e^{\frac{\eta \nu}{\zeta}}-1\right) e^{-\frac{\eta_{*} \nu}{2 \zeta}} d \zeta+O(\eta) \\
\leq & \frac{12 \pi}{\eta_{*}} C_{1} \int_{0}^{\sqrt{\eta}} \max _{\mu}|\tilde{P}| \frac{\zeta^{5}}{\nu^{4}}\left(e^{\frac{\eta \nu}{\zeta}}-1\right) e^{-\frac{\eta_{*} \nu}{2 \zeta}} d \zeta \\
& +\frac{12 \pi}{\eta_{*}} C_{1} \int_{\sqrt{\eta}}^{\infty} \max _{\mu}|\tilde{P}| \frac{\zeta^{5}}{\nu^{4}}\left(e^{\eta c_{1}\left(1+\frac{1}{\sqrt{\eta}}\right)}-1\right) d \zeta+O(\eta)=O(\sqrt{\eta}) \rightarrow 0, \quad \text { as } \eta \rightarrow 0,
\end{aligned}
$$

where $\tilde{P}(\mu, \zeta)=\max \left(|a|,|b|,|c|, \max _{s \geq \eta_{*}}|C[h]|\right)|\mathcal{P}|$. In the last line we have used that there exist positive constants $c_{0}$ and $c_{1}$ s.t. $c_{0}(1+\zeta)<\nu(\zeta)<c_{1}(1+\zeta)$. As to the remaining

$$
\Delta f_{2} \equiv\left|f_{2}(z, \eta)-f_{2}(0,0)\right|=\left|\ln z-(z-1) e^{z} \mathrm{E}_{1}(z)+\frac{1}{2} z\left[z e^{z} \mathrm{E}_{1}(z)-1\right]+\gamma\right|,
$$

we have, as in the case of (24),

$$
\begin{aligned}
& \int_{\mu \zeta<0}\left|\mathcal{P}(\mu, \zeta) b(\mu, \zeta) \Delta f_{2}\right| \frac{\left(1-\mu^{2}\right) \zeta^{2}}{\nu^{3}} d \zeta \leq 2 \pi \int_{0}^{\infty} \frac{\zeta^{4}}{\nu^{3}} \max _{\mu}|\tilde{P}| \int_{0}^{1} \Delta f_{2} d \mu d \zeta \\
& =2 \pi \eta \int_{0}^{\infty} \frac{\zeta^{3}}{\nu^{2}} \max _{\mu}|\tilde{P}|\left(\int_{\frac{\nu \eta}{\zeta}}^{\infty} z^{-2} \Delta f_{2} d z\right) d \zeta \leq 2 \pi \eta \int_{0}^{\infty} \frac{\zeta^{3}}{\nu^{2}} \max _{\mu}|\tilde{P}|\left(\int_{c_{0} \eta}^{\infty} z^{-2} \Delta f_{2} d z\right) d \zeta .
\end{aligned}
$$

Then, using the fact that $\Delta f_{2}$ is $O(z)$ for $z \ll 1$ and $O(\ln z)$ for $z \gg 1$, we see that the first and the second term of the following splitting

$$
\int_{c_{0} \eta}^{\infty} z^{-2} \Delta f_{2} d z=\int_{c_{0} \eta}^{c_{0} \eta_{*}} z^{-2} \Delta f_{2} d z+\int_{c_{0} \eta_{*}}^{\infty} z^{-2} \Delta f_{2} d z
$$

are $O(\ln \eta)$ and $O(1)$, respectively. And finally we obtain

$$
\int_{\mu \zeta<0}\left|\mathcal{P}(\mu, \zeta) b(\mu, \zeta) \Delta f_{2}\right| \frac{\left(1-\mu^{2}\right) \zeta^{2}}{\nu^{3}} d \zeta \leq O(\eta \ln \eta) \rightarrow 0, \quad \text { as } \eta \rightarrow 0 .
$$

This completes the proof of (33), thus that of (16b).

\section{Data of Computations and Measure of Accuracy}

For the check of numerical accuracy, besides the grids S1-S3 and M1-M7 in [18], we have introduced a new grid S4 for $\eta$ space, which is defined by setting $\left(N, N_{\eta}\right)=(200,250)$ in (B.1) of [18] and is twice as fine as the standard spatial grid S1.

The truncation of the $\zeta$ and $\eta$ spaces is justified by confirming the sufficient decay of the velocity distribution function at $\eta=d$ and $\zeta=Z$. Table 3 shows the results in the case of the standard grid (S1,M1), for which $d=44.46$ and $Z=5.0$. The sufficient decay is actually observed. One may think that the decay at $\zeta=Z$ would not be enough for $\phi E, \psi_{A} E$, and $\psi_{B} E$, when compared with the others. Actually, however, a small extension of $Z$ improves a lot. For instance, $\max _{i>1}\left|\phi_{4} E\left(\eta^{(i)}, \cdot, Z\right)\right|$ improves from $2.6 \times 10^{-4}$ with $(\mathrm{S} 1, \mathrm{M} 1)$ to $3.9 \times 10^{-8}$ with $(\mathrm{S} 1, \mathrm{M} 6)$ (remind that $\left.\phi_{4}=\bar{\phi}_{4}+\phi\right)$, where the arrangement of grid points are common between M1 and M6 for $0 \leq \zeta \leq 5.0$ and M6 covers a wider region, i.e., $0 \leq \zeta \leq 5.8$. As will be shown below, the difference of the results between (S1,M1) and $(\mathrm{S} 1, \mathrm{M} 6)$ is negligible, at least at the level of the macroscopic quantities like the slip/jump coefficients.

Although the results in Sect. 5 are obtained by the splitting of solution explained at the beginning of Sect. 4 , we have also solved $\phi_{4}$ and $\psi_{5} \sim \psi_{8}$ directly without the splitting. The results are hardly different from each other. Indeed, the difference of the results between the two manners in the Knudsen-layer functions is less than $7.9 \times 10^{-10}$ in the case of grid $(\mathrm{S} 1, \mathrm{M} 1)$. Therefore, we have examined the grid dependence of the results by the computation 
Table 3 Data for the decay assessment of the velocity distribution function at the edge of the computational region.

\begin{tabular}{cccc}
\hline$F$ & $\max |F(\cdot, \cdot, Z)|$ & $\max |F(d, \cdot, \cdot)|$ & $\max |F|$ \\
\hline $\bar{\phi}_{4} E$ & $4.0 \times 10^{-10}$ & $6.3 \times 10^{-10}$ & 2.0 \\
$\bar{\psi}_{5} E$ & $3.8 \times 10^{-11}$ & $5.3 \times 10^{-13}$ & 1.1 \\
$\bar{\psi}_{6} E$ & $3.1 \times 10^{-11}$ & $4.7 \times 10^{-12}$ & 0.36 \\
$\bar{\psi}_{7} E$ & $4.1 \times 10^{-11}$ & $4.6 \times 10^{-10}$ & 0.95 \\
\hline \hline$F$ & $\max _{i \geq 1}\left|F\left(\eta^{(i)}, \cdot, Z\right)\right|$ & $\max |F(d, \cdot, \cdot)|$ & $\max _{i \geq 1}\left|F\left(\eta^{(i)}, \cdot, \cdot\right)\right|$ \\
\hline$\phi E$ & $2.6 \times 10^{-4}$ & $5.2 \times 10^{-9}$ & $1.7 \times 10^{5}$ \\
$\psi_{A} E$ & $1.3 \times 10^{-5}$ & $8.0 \times 10^{-12}$ & $1.3 \times 10^{5}$ \\
$\psi_{B} E$ & $8.7 \times 10^{-5}$ & $6.5 \times 10^{-11}$ & $2.8 \times 10^{5}$ \\
\hline
\end{tabular}

Table 4 Slip/jump coefficients $c_{4}^{(0)}$ and $b_{5}^{(1)} \sim b_{8}^{(1)}$ obtained by different grids.

\begin{tabular}{cccccc}
\hline Grid & $c_{4}^{(0)}$ & $b_{5}^{(1)}$ & $b_{6}^{(1)}$ & $b_{7}^{(1)}$ & $b_{8}^{(1)}$ \\
\hline (S1,M1) & 4.6180185 & -0.6601218 & 0.2438061 & 0.4472751 & -0.2335314 \\
(S1,M2) & 4.6180185 & -0.6601218 & 0.2438061 & 0.4472751 & -0.2335314 \\
(S1,M3) & 4.6180492 & -0.6601221 & 0.2438051 & 0.4472728 & -0.2335330 \\
(S1,M4) & 4.6180143 & -0.6601211 & 0.2438071 & 0.4472741 & -0.2335313 \\
(S1,M5) & 4.6180185 & -0.6601218 & 0.2438061 & 0.4472751 & -0.2335314 \\
(S1,M6) & 4.6180221 & -0.6601220 & 0.2438061 & 0.4472750 & -0.2335315 \\
(S1,M7) & 4.6178471 & -0.6601224 & 0.2438091 & 0.4472922 & -0.2335224 \\
(S2,M1) & 4.6180153 & -0.6601231 & 0.2438053 & 0.4472744 & -0.2335334 \\
(S3,M1) & 4.6180185 & -0.6601218 & 0.2438061 & 0.4472750 & -0.2335314 \\
(S4,M1) & 4.6180148 & -0.6601233 & 0.2438052 & 0.4472743 & -0.2335337 \\
(S4,M3) & 4.6180455 & -0.6601236 & 0.2438041 & 0.4472720 & -0.2335353 \\
\hline
\end{tabular}

without splitting only. The grid dependence of the computed slip/jump coefficients is shown in Table 4 . The grid in $\zeta$-space most affects the results, especially for $c_{4}^{(0)}, b_{7}^{(1)}$, and $b_{8}^{(1)}$ [compare the results by $(\mathrm{S} 1, \mathrm{M} 1),(\mathrm{S} 1, \mathrm{M} 3)$, and $(\mathrm{S} 1, \mathrm{M} 7)$, where $\mathrm{M} 3$ (or M1) is the grid about twice (or 3/2) as many points as M7]. The accuracy down to the fourth or fifth decimal place is expected from the table. M2 is the grid that refines M1 only in the range that $M$ is small. The comparison between $(\mathrm{S} 1, \mathrm{M} 1)$ and $(\mathrm{S} 1, \mathrm{M} 2)$ in the table shows that $\mathrm{M} 1$ is fine enough for small $M$. The comparisons among (S1,M1), (S3,M1), and $(\mathrm{S} 1, \mathrm{M} 6)$ in the table show that the error due to the truncation of the $\zeta$ and $\eta$ spaces is almost negligible.

The accuracy of the collision integral computation has already been assessed in [18] by checking the identities $C\left[\left(1, \mu \zeta, \zeta^{2}\right) E\right]=\left(1, \mu \zeta, \zeta^{2}\right) \nu E$ for $C=E \mathcal{C} E^{-1}$ and $C[E]=\nu E$ for $C=E \mathcal{C}^{S} E^{-1}$. With the standard grid M1, these identities are confirmed to hold within the error of $9.1 \times 10^{-8}, 1.7 \times 10^{-8}, 6.6 \times 10^{-8}$, and $8.9 \times 10^{-9}$ respectively, while the maximum values of $\left(1, \mu \zeta, \zeta^{2}\right) \nu E$ are $0.13,0.064$, and 0.062 respectively. [18]

The mass, momentum, and energy balances offer another measure of accuracy. They are the following identities that are obtained from (4a) and (5a) by the integration in molecular velocity space after multiplying the collision invariants:

$$
\begin{aligned}
& \left\langle\mu \zeta \phi_{4}\right\rangle=-2 \int_{\eta}^{\infty} Y_{2}^{(1)}(z) d z,\left\langle\mu^{2} \zeta^{2} \phi_{4}\right\rangle=-\frac{3}{2} \int_{\eta}^{\infty}\left[\Omega_{1}^{(0)}(z)+\Theta_{1}^{(0)}(z)\right] d z \\
& \left\langle\mu \zeta\left(\zeta^{2}-\frac{5}{2}\right) \phi_{4}\right\rangle=-2 \int_{\eta}^{\infty} H_{2}^{(1)}(z) d z \\
& \left\langle\mu\left(1-\mu^{2}\right) \zeta^{3} \psi_{7}\right\rangle=\frac{3}{2} \int_{\eta}^{\infty}\left[\Omega_{1}^{(0)}(z)+\Theta_{1}^{(0)}(z)\right] d z \\
& \left\langle\mu\left(1-\mu^{2}\right) \zeta^{3} \psi_{i}\right\rangle_{+}=-\left\langle\mu\left(1-\mu^{2}\right) \zeta^{3} \psi_{i}\right\rangle_{-}, \quad(i=5,6,8) .
\end{aligned}
$$


Here $\langle\cdot\rangle_{ \pm}$is the half-range integral with respect to the molecular velocity defined by $\langle f(\boldsymbol{\zeta})\rangle_{ \pm}=$ $\langle f(\zeta) \chi[0,1]( \pm \mu)\rangle$. With the standard grid (S1,M1), the identities in (38a) hold within the error of $6.0 \times 10^{-7}, 3.2 \times 10^{-6}$, and $5.0 \times 10^{-6}$, while the maxima of their left-hand side are $0.96,0.29$, and 2.6. In the case of (38b), the error is within $5.1 \times 10^{-6}$, while the maximum of the 1.h.s. is 0.29 . In the case of (38c), the error is within $7.6 \times 10^{-7}, 3.2 \times 10^{-6}$, or $7.2 \times 10^{-7}$ $(i=5,6$, or 8$)$, while the maximum of the l.h.s. are $0.27,0.12$, and $0.13(i=5,6$, or 8$)$.

\section{References}

1. Hilbert, D.: Begründung der kinetischen Gastheorie. Math. Ann. 72, 562-577 (1912)

2. Chapman, S., Cowling, T.G.: The Mathematical Theory of Non-uniform Gases, Cambridge University Press, 3rd ed., Cambridge (1991)

3. Grad, H.: Asymptotic theory of the Boltzmann equation. Phys. Fluids 6, 147-181 (1963)

4. Sone, Y.: Asymptotic theory of flow of rarefied gas over a smooth boundary I. In: Trilling, L., Wachman, H.Y. (eds.) Rarefied Gas Dynamics, vol. I, pp. 243-253. Academic Press, New York (1969)

5. Sone, Y.: Asymptotic theory of flow of rarefied gas over a smooth boundary II. In: Dini, D. (ed.) Rarefied Gas Dynamics, vol. II, pp. 737-749. Edtrice Tecnico Scientifica, Pisa (1971)

6. Sone, Y.: Molecular Gas Dynamics. Birkhäuser, Boston (2007). Supplementary Notes and Errata is available from Kyoto University Research Information Repository (http://hdl.handle.net/2433/66098)

7. Golse, F., Levermore, C.D.: Stokes-Fourier and acoustic limits for the Boltzmann equation: convergence proofs. Comm. Pure Appl. Math. 55, 336-393 (2002)

8. Golse, F., Saint-Raymond, L.: The Navier-Stokes limit of the Boltzmann equation for bounded collision kernels. Invent. Math. 155, 81-161 (2004)

9. Yu, S.-H.: Hydrodynamic limits with shock waves of the Boltzmann equation. Comm. Pure Appl. Math. 58, 409-443 (2005)

10. Takata, S., Hattori, M.: Asymptotic theory for the time-dependent behavior of a slightly rarefied gas over a smooth solid boundary. J. Stat. Phys. 147, 1182-1215 (2012)

11. Nassios, J., Sader, J.E.: Asymptotic analysis of the Boltzmann-BGK equation for oscillatory flows. J. Fluid Mech. 708, 197-249 (2012)

12. Inaba, M., Yano, T., Watanabe, M.: Linear theory of sound waves with evaporation and condensation. Fluid Dyn. Res. 44, 025506 (2012)

13. Masmoudi, N., Saint-Raymond, L.: From the Boltzmann equation to the Stokes-Fourier system in a bounded domain. Comm. Pure Appl. Math. 56, 1263-1293 (2003)

14. Jiang, N., Levermore, C.D., Masmoudi, N.: Remarks on the acoustic limit for the Boltzmann equation. Comm. P. D. E. 35, 1590-1609 (2010)

15. Golse, F.: From the Boltzmann equation to the Euler equations in the presence of boundaries. Comput. Math. Appl. 65, 815-830 (2013)

16. Bhatnagar, P.L., Gross, E.P., Krook, M.: A model for collision processes in gases. I. small amplitude processes in charged and neutral one-component systems. Phys. Rev. 94, 511-525 (1954)

17. Welander, P.: On the temperature jump in a rarefied gas. Ark. Fys. 7, 507-553 (1954)

18. Hattori, M., Takata, S.: Second-order Knudsen-layer analysis for the generalized slipflow theory I. Bulletin of the Institute of Mathematics, Academia Sinica (New Series) (submitted)

19. Sone, Y., Ohwada, T., Aoki, K.: Temperature jump and Knudsen layer in a rarefied gas over a plane wall: numerical analysis of the linearized Boltzmann equation for hardsphere molecules. Phys. Fluids A 1, 363-370 (1989)

20. Ohwada, T., Sone, Y., Aoki, K.: Numerical analysis of the shear and thermal creep flows of a rarefied gas over a plane wall on the basis of the linearized Boltzmann equation for hard-sphere molecules. Phys. Fluids A 1, 1588-1599 (1989)

21. Ohwada, T., Sone, Y.: Analysis of thermal stress slip flow and negative thermophoresis using the Boltzmann equation for hard-sphere molecules. Eur. J. Mech. B/Fluids 11, 389-414 (1992) 
22. Takata, S., Aoki, K., Hattori, M., Hadjiconstantinou, N.G.: Parabolic temperature profile and second-order temperature jump of a slightly rarefied gas in an unsteady twosurface problem. Phys. Fluids 24, 032002 (2012)

23. Takata, S.: Symmetry of the linearized Boltzmann equation and its application. J. Stat. Phys. 136, 751-784 (2009)

24. Takata, S., Funagane, H.: Poiseuille and thermal transpiration flows of a highly rarefied gas: over-concentration in the velocity distribution function. J. Fluid Mech. 669, 242$259(2011)$

25. Takata, S., Funagane, H.: Singular behaviour of a rarefied gas on a planar boundary. J. Fluid Mech. 717, 30-47 (2013)

26. Bardos, C., Caflisch, R.E., Nicolaenko, B.: The Milne and Kramers problems for the Boltzmann equation of a hard sphere gas. Comm. Pure Appl. Math. 39, 323-352 (1986)

27. Kennard, E.H.: Kinetic Theory of Gases, McGraw-Hill, New York (1938)

28. Sone, Y.: Flow induced by thermal stress in rarefied gas. Phys. Fluids 15, 1418-1423 (1972)

29. Sone, Y., Takata, S.: Discontinuity of the velocity distribution function in a rarefied gas around a convex body and the $\mathrm{S}$ layer at the bottom of the Knudsen layer. Transp. Theory Stat. Phys. 21, 501-530 (1992) 